\title{
Constructing fifteen infinite classes of nonregular bipartite integral graphs*
}

\author{
Ligong Wang ${ }^{1, \dagger}$ and Cornelis Hoede ${ }^{2}$ \\ ${ }^{1}$ Department of Applied Mathematics, School of Science, \\ Northwestern Polytechnical University, Xi'an, Shaanxi 710072, P. R. China. \\ ligongwangnpu@yahoo.com.cn \\ ${ }^{2}$ Department of Applied Mathematics, \\ Faculty of Electrical Engineering, Mathematics and Computer Science, \\ University of Twente, P.O. Box 217, 7500AE Enschede, The Netherlands. \\ hoede@math. utwente.nl \\ Submitted: Oct 5, 2007; Accepted: Dec 16, 2007; Published: Jan 1, 2008 \\ Mathematics Subject Classifications: 05C50, 11D09, 11D41
}

\begin{abstract}
A graph is called integral if all its eigenvalues (of the adjacency matrix) are integers. In this paper, the graphs $S_{1}(t)=K_{1, t}, S_{2}(n, t), S_{3}(m, n, t), S_{4}(m, n, p, q)$, $S_{5}(m, n), S_{6}(m, n, t), S_{8}(m, n), S_{9}(m, n, p, q), \quad S_{10}(n), S_{13}(m, n), \quad S_{17}(m, n, p, q)$, $S_{18}(n, p, q, t), S_{19}(m, n, p, t), S_{20}(n, p, q)$ and $S_{21}(m, t)$ are defined. We construct the fifteen classes of larger graphs from the known 15 smaller integral graphs $S_{1}-S_{6}$, $S_{8}-S_{10}, S_{13}, S_{17}-S_{21}$ (see also Figures 4 and 5, Balińska and Simić, Discrete Math. 236(2001) 13-24). These classes consist of nonregular and bipartite graphs. Their spectra and characteristic polynomials are obtained from matrix theory. We obtain their integral property by using number theory and computer search. All these classes are infinite. They are different from those in the literature. It is proved that the problem of finding such integral graphs is equivalent to solving Diophantine equations. We believe that it is useful for constructing other integral graphs. The discovery of these integral graphs is a new contribution to the search of integral graphs. Finally, we propose several open problems for further study.
\end{abstract}

\section{Introduction}

We use $G$ to denote a simple graph with vertex set $V(G)=\left\{v_{1}, v_{2}, \ldots, v_{n}\right\}$ and edge set $E(G)$. The adjacency matrix $A=A(G)=\left[a_{i j}\right]$ of $G$ is an $n \times n$ symmetric matrix of 0 's

\footnotetext{
* Supported by National Science Foundation of China and Natural Science Basic Research Plan in Shaanxi Province of China.

${ }^{\dagger}$ Corresponding author.
} 
and 1's with $a_{i j}=1$ if and only if $v_{i}$ and $v_{j}$ are joined by an edge. The characteristic polynomial of $G$ is the polynomial $P(G)=P(G, x)=\operatorname{det}\left(x I_{n}-A\right)$, where and in the sequel $I_{n}$ always denotes the $n \times n$ identity matrix. The spectrum of $A(G)$ is also called the spectrum of $G$ and denoted by $\operatorname{Spec}(G)([5])$.

A graph $G$ is called integral if all eigenvalues of the characteristic polynomial $P(G, x)$ of $G$ are integers. The research on integral graphs was initiated by Harary and Schwenk [7]. In general, the problem of characterizing integral graphs seems to be very difficult. Thus, it makes sense to restrict our investigations to some interesting families of graphs. So far, there are many results for some particular classes of integral graphs [1]. For all other facts or terminology on graph spectra, see [5].

In [9] we successfully constructed integral trees of diameters 4 and 6 by identifying the centers of two trees. In $[10,11]$ we investigated the structures of some classes of graphs and deduce their characteristic polynomials by spectral graph theory. Integral graphs in these classes were given by using number theory and computer search. In this paper, a new method of constructing fifteen infinite classes of integral graphs is presented. In getting the results we proceed as follows: firstly, we give the construction of the (infinite) families of new graphs from the 15 finite classes of integral graphs identified by Balinska and Simić [2], then calculate their characteristic polynomials (Theorem 3.2) by using matrix theory, and then, by making use of number theory (Diophantine equations) and computer search, we obtain fifteen infinite classes of integral graphs in these classes. These classes are connected nonregular and bipartite graphs except for several disconnected graphs for which one or several of their parameters are taken zero. Finally, we propose several open problems for further study.

\section{Some facts in matrix theory and number theory}

In this section, we shall give a useful property of matrices and some facts in number theory.

First of all, we give the following notations. All other notations and terminology on matrices can be found in [6].

(1) $R$ denotes the set of real numbers.

(2) $R^{m \times n}$ denotes the set of $m \times n$ matrices whose entries are in $R$.

(3) $A^{T}$ denotes the transpose of the matrix $A$.

(4) $J_{m \times n}$ and $0_{m \times n}$ denotes the $m \times n$ all 1 and all 0 matrix, respectively.

Lemma 2.1. ([6], page 181) Let $A=\left[\begin{array}{cc}A_{0} & A_{1} \\ A_{1} & A_{0}\end{array}\right]$, where $A_{k} \in R^{r \times r}, k=0,1$. Then the eigenvalues of $A$ are those of $A_{0}+A_{1}$ together with those of $A_{0}-A_{1}$.

Secondly, we shall give some facts in number theory. All other notations and terminology on number theory can be found in $[4,8]$.

Let $d$ be a positive integer but not a perfect square, let $m \neq 0$ be an integer. We shall study the Diophantine equation

$$
x^{2}-d y^{2}=m \text {. }
$$


If $x_{1}, y_{1}$ is a solution of (1), for convenience, then $x_{1}+y_{1} \sqrt{d}$ is also called a solution of Eq.(1). Let $s+t \sqrt{d}$ be any solution of the Pell equation

$$
x^{2}-d y^{2}=1
$$

Clearly, $\left(x_{1}+y_{1} \sqrt{d}\right)(s+t \sqrt{d})=x_{1} s+y_{1} t d+\left(y_{1} s+x_{1} t\right) \sqrt{d}$ is also a solution of Eq.(1). This solution and $x_{1}+y_{1} \sqrt{d}$ are called associate. If two solutions $x_{1}+y_{1} \sqrt{d}$ and $x_{2}+y_{2} \sqrt{d}$ of Eq.(1) are associate, then we denote them by $x_{1}+y_{1} \sqrt{d} \sim x_{2}+y_{2} \sqrt{d}$. It is easy to verify that the associate relation $\sim$ is an equivalence relation. Hence, if Eq.(1) has solutions, then all the solutions can be classified by the associate relation. Any two solutions in the same associate class are associate each other, any two solutions not in the same class are not associate.

The following Lemmas 2.2, 2.3, 2.4 and 2.5 can be found in [4].

Lemma 2.2. A necessary and sufficient condition for two solutions $x_{1}+y_{1} \sqrt{d}$ and $x_{2}+$ $y_{2} \sqrt{d}$ of Eq.(1) ( $m$ fixed) to be in the same associate class $K$ is that

$$
x_{1} x_{2}-d y_{1} y_{2} \equiv 0(\bmod |m|) \quad \text { and } \quad y_{1} x_{2}-x_{1} y_{2} \equiv 0(\bmod |m|) .
$$

Let $x_{1}+y_{1} \sqrt{d}$ be any solution of Eq.(1). By Lemma 2.2, we see that $-\left(x_{1}+y_{1} \sqrt{d}\right) \sim$ $x_{1}+y_{1} \sqrt{d},-\left(x_{1}-y_{1} \sqrt{d}\right) \sim x_{1}-y_{1} \sqrt{d}$. Let $K$ and $K^{\prime}$ be two associate classes of solutions of Eq. (1) such that for any solution $x+y \sqrt{d} \in K$, it follows $x-y \sqrt{d} \in K^{\prime}$. Then also the converse is true. Hence, $K$ and $K^{\prime}$ are called conjugate classes. If $K=K^{\prime}$, then this class is called an ambiguous class. Let $u_{0}+v_{0} \sqrt{d}$ be the fundamental solution of the associate class $K$, i.e. $v_{0}$ is positive and has the smallest value in the class $K$. If the class $K$ is ambiguous, we can assume that $u_{0} \geq 0$.

Lemma 2.3. Let $K$ be any associate class of solutions of Eq.(1), and let $u_{0}+v_{0} \sqrt{d}$ be the fundamental solution of the associate class $K$. Let $x_{0}+y_{0} \sqrt{d}$ be the fundamental solution of the Pell equation (2). Then

$$
\begin{gathered}
0 \leq v_{0} \leq \begin{cases}\frac{y_{0} \sqrt{m}}{\sqrt{2\left(x_{0}+1\right)},}, & \text { if } m>0, \\
\frac{y_{0} \sqrt{-m}}{\sqrt{2\left(x_{0}-1\right)}}, & \text { if } m<0 .\end{cases} \\
0 \leq\left|u_{0}\right| \leq \begin{cases}\sqrt{\frac{1}{2}\left(x_{0}+1\right) m}, & \text { if } m>0, \\
\sqrt{\frac{1}{2}\left(x_{0}-1\right)(-m)}, & \text { if } m<0 .\end{cases}
\end{gathered}
$$

\section{Lemma 2.4.}

(1) Let $d$ be a positive integer but not a perfect square, $m \neq 0$, and let $m$ be an integer. Then there are only finitely many associate classes for Eq.(1), and the fundamental solutions of all these classes can be found from (3) and (4) by a finite procedure. 
(2) Let $K$ be an associate class of solutions of Eq. (1), and let $u_{0}+v_{0} \sqrt{d}$ be the fundamental solution of the associate class $K$. Then all solutions of the class $K$ are given by

$$
x+y \sqrt{d}= \pm\left(u_{0}+v_{0} \sqrt{d}\right)\left(x_{0}+y_{0} \sqrt{d}\right)^{n},
$$

where $n$ is an integer, and $x_{0}+y_{0} \sqrt{d}$ is the fundamental solution of Eq.(2).

(3) If $u_{0}$ and $v_{0}$ satisfy (3) and (4) but are not solutions of Eq.(1), then there is no solution for Eq.(1).

Lemma 2.5. Let $d(>1)$ be a positive integer that is not a perfect square. Then there exist solutions for the Pell equation (2), and all the positive integral solutions $x_{k}, y_{k}$ of Eq.(2) are given by

$$
x_{k}+y_{k} \sqrt{d}=\varepsilon^{k}, \quad k=1,2, \ldots,
$$

where $\varepsilon=x_{0}+y_{0} \sqrt{d}$ is the fundamental solution of Eq.(2). Put $\bar{\varepsilon}=x_{0}-y_{0} \sqrt{d}$. Then we have $\varepsilon \bar{\varepsilon}=1$ and

$$
x_{k}=\frac{\varepsilon^{k}+\bar{\varepsilon}^{k}}{2}, \quad y_{k}=\frac{\varepsilon^{k}-\bar{\varepsilon}^{k}}{2 \sqrt{d}}, \quad k=1,2, \ldots
$$

The following Lemmas 2.6, 2.9, 2.11 and Lemmas 2.7, 2.8 can be found in [8] and [4], respectively.

Lemma 2.6. Let $u, v$ be the fundamental solution of Eq.(2), where $d(>1)$ is a positive integer but not a perfect square. Then the Pell equation

$$
x^{2}-d y^{2}=-1
$$

has solutions if and only if there exist positive integer solutions $s$ and $t$ for the equations

$$
s^{2}+d t^{2}=u, \quad 2 s t=v,
$$

such that moreover s and $t$ are the fundamental solution of Eq.(7).

Lemma 2.7. Suppose the Pell equation (7) is solvable. Let $\rho=x_{0}+y_{0} \sqrt{d}$ be the fundamental solution of Eq.(7), where $d(>1)$ is a positive integer but not a perfect square. Then the following holds.

(1) All positive integral solutions $x_{k}, y_{k}$ of Eq.(7) are given by

$$
x_{k}+y_{k} \sqrt{d}=\rho^{k}, \quad k=1,3,5, \ldots
$$

(2) All positive integral solutions $x_{k}, y_{k}$ of $E q$. (2) are given by relation (8), $k=2,4, \ldots$.

(3) Let $\bar{\rho}=x_{0}-y_{0} \sqrt{d}$, then $\rho \bar{\rho}=-1$, and the solutions $x_{k}, y_{k}$ in (1) and (2) can be given by

$$
x_{k}=\frac{\rho^{k}+\bar{\rho}^{k}}{2}, \quad y_{k}=\frac{\rho^{k}-\bar{\rho}^{k}}{2 \sqrt{d}}, \quad k=1,2, \ldots
$$




\section{Lemma 2.8.}

(1) If there is a solution for Eq.(1), where $m \neq 0$ is integer and $d(>1)$ is a positive integer but not a perfect square, then Eq.(1) has infinitely many solutions.

(2) Let $x_{1}, y_{1}$ be the fundamental solution of the Diophantine equation

$$
x^{2}-d y^{2}=4
$$

where $d(>1)$ is a positive integer but not a perfect square. Then all positive integral solutions $x_{k}, y_{k}$ of Eq.(10) are given by

$$
\frac{x_{k}+y_{k} \sqrt{d}}{2}=\left(\frac{x_{1}+y_{1} \sqrt{d}}{2}\right)^{k}, \quad k=1,2, \ldots
$$

In the following symbol $(a, b)=d$ denotes the greatest common divisor $d$ of integers $\mathrm{a}$, b, while $a \mid b(a \nmid b)$ means that $a$ divides $b$ ( $a$ does not divide $b$ ) .

Lemma 2.9. Let $m$ be a positive integer. If $2 \nmid m$ or $4 \mid m$, then there exist positive integral solutions for the Diophantine equation

$$
x^{2}-y^{2}=m
$$

Remark 2.10. We can give a method for finding the solutions of Eq.(12). Suppose that $m=m_{1} m_{2}$. Let $x-y=m_{1}, x+y=m_{2}$ and $2 \mid\left(m_{1}+m_{2}\right)$. Then the solutions of Eq. (12) can be found easily (see [8]).

Lemma 2.11. If $x>0, y>0, z>0,(x, y)=1$ and $2 \mid y$, then all positive integral solutions of the Diophantine equation $x^{2}+y^{2}=z^{2}$ are given by

$$
x=r^{2}-s^{2}, y=2 r s, z=r^{2}+s^{2},
$$

where $(r, s)=1, r>s>0$ and $2 \nmid r+s$.

\section{The characteristic polynomials of some classes of graphs}

In this section, we investigate the structures of the nonregular bipartite integral graphs in [2]. Fifteen new classes of larger graphs are constructed based on the structures of 15 ones of the 21 smaller integral graphs in Figures 4 and 5 of [2].

Theorem 3.1. ([2]) The graphs in Figures 1 and 2 are nonregular bipartite integral graphs with maximum degree four. (The graphs in Figure 1 are integral graphs with number of vertices up to 16.) 

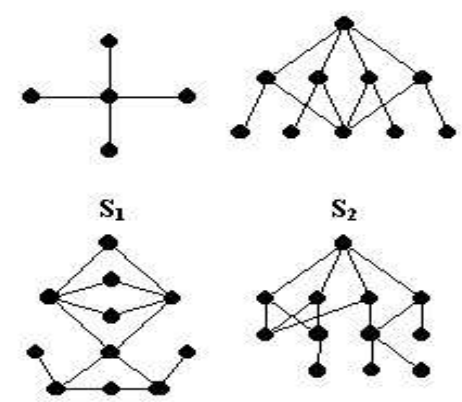

$S_{7}$

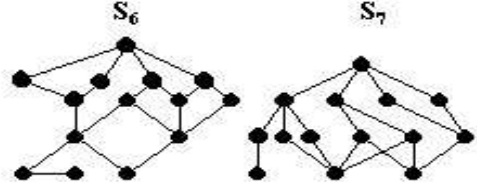

$\mathbf{S}_{\mathbf{1}}$

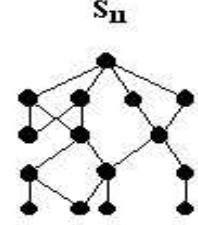

$\mathbf{S}_{16}$

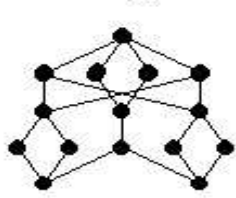

$S_{17}$

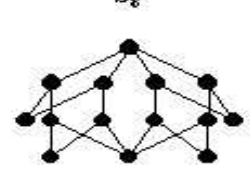

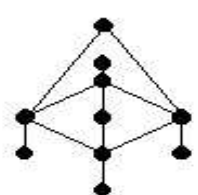

$\mathbf{S}_{3}$

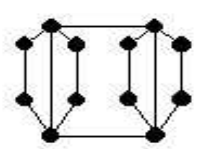

$\mathbf{S}_{\mathbf{8}}$

$S_{13}$

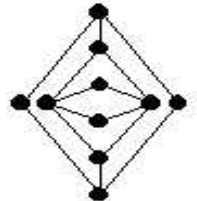

$\mathbf{S}_{4}$

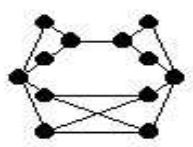

$\mathbf{S}_{9}$

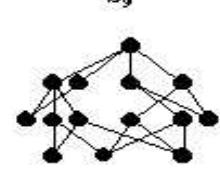

$S_{14}$

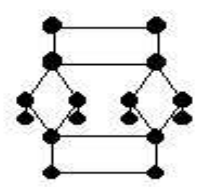

$S_{18}$

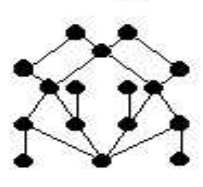

$S_{19}$

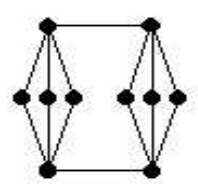

$\mathbf{S}_{5}$

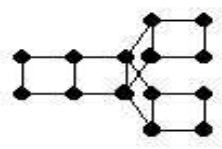

$\mathbf{S}_{10}$

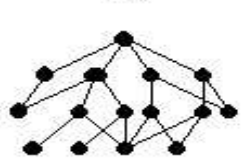

$S_{15}$

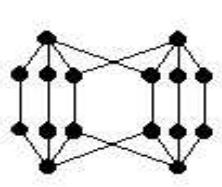

$S_{20}$

Figure 1: Nonregular bipartite integral graphs with maximum degree 4 and at most 16 vertices.

We can generalize the result of Theorem 3.1 and construct fifteen types of graphs from 15 smaller integral graphs $S_{1}-S_{6}, S_{8}-S_{10}, S_{13}, S_{17}-S_{21}$ in Figures 1 and 2. The following Theorem 3.2 on their characteristic polynomials is obtained from matrix theory.

Theorem 3.2. Let $m, n, p, q$ and $t$ be nonnegative integers. Then the characteristic polynomials of the fifteen types of graphs in Figures 3 and 4 are as follows:

(1) (see [5]) $P\left(K_{1, t}, x\right)=x^{t-1}\left(x^{2}-t\right),(t \geq 0)$.

(2) $P\left(S_{2}(n, t), x\right)=x^{n(t-1)+2}\left(x^{2}-t\right)^{n-1}\left[x^{2}-(2 n+t)\right],(n \geq 1, t \geq 0)$.

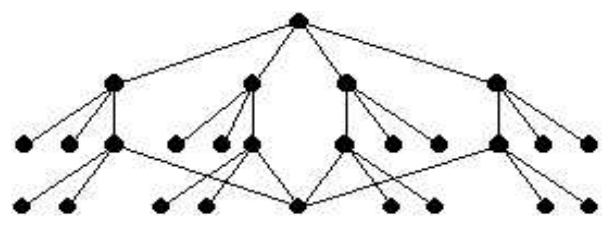

$S_{21}$

Figure 2: A nonregular bipartite integral graph with maximum degree 4 and 26 vertices. 
(3) $P\left(S_{3}(m, n, t), x\right)=x^{m+n+4(t-1)}\left(x^{2}-t\right)^{2}\left[x^{4}-2(m+n+t+2) x^{2}+(2 m+t)(2 n+t)\right]$, $(m \geq 1, n \geq 1, t \geq 0)$.

(3.1) $P\left(S_{3}(n, n, t), x\right)=x^{2 n+4(t-1)}\left(x^{2}-t\right)^{2}\left(x^{2}+2 x-2 n-t\right)\left(x^{2}+2 x-2 n-t\right),(n \geq 1$, $t \geq 0)$.

(3.2) $P\left(S_{3}(m, n, 0), x\right)=x^{m+n}\left[x^{4}-2(m+n+2) x^{2}+4 m n\right],(m \geq 1, n \geq 1)$.

(4) $P\left(S_{4}(m, n, p, q), x\right)=x^{m p+n+2 q-2}\left(x^{2}-2 m\right)^{p-1}\left(x^{2}-p q\right)\left[x^{4}-(2 m+2 n+4 q+p q) x^{2}+\right.$ $4 m n+8 m q+2 n p q],(m \geq 0, n \geq 0, p \geq 1, q \geq 1)$.

(4.1) $P\left(S_{4}(n, n, p, q), x\right)=x^{n(p+1)+2 q-2}\left(x^{2}-2 n\right)^{p}\left(x^{2}-p q\right)\left[x^{2}-(2 n+4 q+p q)\right],(n \geq 0$, $p \geq 1, q \geq 1)$.

(4.2) $P\left(S_{4}(n, n, p, p), x\right)=x^{n(p+1)+2 p-2}\left(x^{2}-2 n\right)^{p}(x+p)(x-p)\left[x^{2}-\left(2 n+p^{2}+4 p\right)\right],(n \geq 1$, $p \geq 1)$.

(4.3) $P\left(S_{4}(2,2, p, p), x\right)=x^{4 p}(x+p+2)(x+p)(x+2)^{p}(x-2)^{p}(x-p)(x-p-2),(p \geq 1)$.

(4.4) $P\left(S_{4}(0, n, p, q), x\right)=x^{n+2 p+2 q-4}\left(x^{2}-p q\right)\left[x^{4}-(2 n+4 q+p q) x^{2}+2 n p q\right],(n \geq 0$, $p \geq 1, q \geq 1)$.

(4.5) $P\left(S_{4}(m, 0, p, q), x\right)=x^{m p+2 q-2}\left(x^{2}-2 m\right)^{p-1}\left(x^{2}-p q\right)\left[x^{4}-(2 m+4 q+p q) x^{2}+8 m q\right]$, $(m \geq 0, p \geq 1, q \geq 1)$.

(5) $P\left(S_{5}(m, n), x\right)=x^{m+n-2}(x+1)(x-1)\left[x^{4}-(2 m+2 n+1) x^{2}+4 m n\right],(m \geq 0, n \geq 0)$.

(5.1) $P\left(S_{5}(n, n), x\right)=x^{2 n-2}(x+1)(x-1)\left(x^{2}+x-2 n\right)\left(x^{2}-x-2 n\right),(n \geq 0)$.

(5.2) $P\left(S_{5}(0, n), x\right)=P\left(S_{5}(n, 0), x\right)=x^{n}(x+1)(x-1)\left[x^{2}-(2 n+1)\right],(n \geq 0)$.

(6) $P\left(S_{6}(m, n, t), x\right)=x^{n(t-1)+m+2}\left(x^{2}-t\right)^{n-1}\left[x^{4}-(2 m+2 n+t+2) x^{2}+2 n(2 m+1)+\right.$ $2 t(m+1)],(m \geq 0, n \geq 1, t \geq 0)$ or $(m \geq 0, n=t=0)$.

(6.1) $P\left(S_{6}(m, 0,0), x\right)=P\left(K_{2, m+1} \cup K_{1}, x\right)=x^{m+2}\left[x^{2}-(2 m+2)\right],(m \geq 1)$.

(6.2) $P\left(S_{6}(0, n, t), x\right)=x^{n(t-1)+2}\left(x^{2}-t\right)^{n-1}\left[x^{4}-(2 n+t+2) x^{2}+2 n+2 t\right],(n \geq 1, t \geq 0)$.

(6.3) $P\left(S_{6}(m, n, 0), x\right)=x^{n+m}\left[x^{4}-(2 m+2 n+2) x^{2}+2 n(2 m+1)\right],(m \geq 0, n \geq 0)$.

(6.4) $P\left(S_{6}(m, 1, t), x\right)=x^{m+t+1}\left[x^{4}-(2 m+t+4) x^{2}+2(2 m+1)+2 t(m+1)\right],(m \geq 0$, $t \geq 0)$.

(6.5) $P\left(S_{6}(n-1, n, 1), x\right)=x^{n+1}(x+1)^{n-1}(x-1)^{n-1}\left(x^{2}+x-2 n\right)\left(x^{2}-x-2 n\right),(n \geq 1)$.

(6.6) $P\left(S_{6}(n+1, n, 1), x\right)=x^{n+3}(x+1)^{n-1}(x-1)^{n-1}\left(x^{2}+x-2 n-2\right)\left(x^{2}-x-2 n-2\right)$, $(n \geq 0)$.

(6.7) $P\left(S_{6}(n+1, n, 9), x\right)=x^{9 n+3}(x+3)^{n-1}(x-3)^{n-1}\left(x^{2}+x-2 n-6\right)\left(x^{2}-x-2 n-6\right)$, $(n \geq 1)$. 
(7) $P\left(S_{8}(m, n), x\right)=(x+1)^{m+n-2}(x-1)^{m+n-2}\left[x^{4}-4 x^{3}-(m+n-5) x^{2}+(2 m+2 n-\right.$ 2) $x+m n-m-n]\left[x^{4}+4 x^{3}-(m+n-5) x^{2}-(2 m+2 n-2) x+m n-m-n\right]$, $(m \geq 0, n \geq 0)$.

(7.1) $P\left(S_{8}(n, n), x\right)=(x+1)^{2 n-2}(x-1)^{2 n-2}\left(x^{2}+x-n\right)\left(x^{2}-x-n\right)\left(x^{2}+3 x-n+2\right)\left(x^{2}-\right.$ $3 x-n+2),(n \geq 0)$.

(8) $P\left(S_{9}(m, n, p, q), x\right)=x^{m+n+p+q-2}\left[x^{6}-(2 m+n+2 p+q+n q+1) x^{4}+(m+n+\right.$ $m n+p+4 m p+2 n p+q+2 m q+2 n q+2 m n q+p q+2 n p q) x^{2}-(m p+n p+2 m n p+$ $m q+n q+2 m n q+2 m p q+2 n p q+4 m p q)],(m \geq 1, n \geq 1 p \geq 1, q \geq 1)$.

(8.1) $P\left(S_{9}(n, n, n, n), x\right)=x^{4 n-2}\left(x^{2}-2 n\right)^{2}(x+n+1)(x-n-1),(n \geq 1)$.

(9) $P\left(S_{10}(n), x\right)=x^{2(n-1)}(x+2)^{n-1}(x+1)(x-1)(x-2)^{n-1}\left(x^{2}+2 x-n\right)\left(x^{2}-2 x-n\right)$, $(n \geq 0)$.

(10) $P\left(S_{13}(m, n), x\right)=x^{2}(x+1)^{n(m-1)}(x-1)^{n(m-1)}\left(x^{2}+x-m\right)^{n-1}\left[x^{2}+x-m(n+1)\right]\left(x^{2}-\right.$ $x-m)^{n-1}\left[x^{2}-x-m(n+1)\right],(m \geq 1, n \geq 1)$.

(11) $P\left(S_{17}(m, n, p, q), x\right)=x^{m q+p+n-1}\left(x^{2}-2 m\right)^{q-1}\left\{x^{6}-(2 m+2 n+p+q+p q+1) x^{4}+\right.$ $\left[m(2+4 n+2 p+q+p q)+n+p+n p+2 n q+2 p q+2 n p q+p q^{2}\right] x^{2}-[2 m(n+p+$ $n p+n q+p q+n p q)+2 n p q(q+1)]\},(m \geq 1, n \geq 1, p \geq 1, q \geq 1)$.

(11.1) $P\left(S_{17}(n, n, n, n), x\right)=x^{n^{2}+2 n-1}(x+n+1)(x-n-1)\left(x^{2}-2 n\right)^{n+1},(n \geq 1)$.

(11.2) $P\left(S_{17}(n, n, p, q), x\right)=x^{n q+n+p-1}\left(x^{2}-2 n\right)^{q}\left\{x^{4}-[2 n+(p+1)(q+1)] x^{2}+(q+1)[n(p+\right.$ 1) $+p(q+1)]\},(n \geq 1, p \geq 1, q \geq 1)$.

(11.3) $P\left(S_{17}(n, n, 1, q), x\right)=x^{n q+n}\left(x^{2}-2 n\right)^{q}\left(x^{2}-q-1\right)\left(x^{2}-2 n-q-1\right),(n \geq 1, q \geq 1)$.

(11.4) $P\left(S_{17}(m, n, m, n), x\right)=x^{m n+m+n-1}\left(x^{2}-2 m\right)^{n-1}\left(x^{2}-m-n\right)\left[x^{4}-(2 m+2 n+m n+\right.$ 1) $\left.x^{2}+2 m(n+1)^{2}\right],(m \geq 1, n \geq 1)$.

(11.5) $P\left(S_{17}(2, n, 2, n), x\right)=x^{3 n+1}(x+2)^{n-1}(x-2)^{n-1}\left(x^{2}-n-2\right)\left(x^{2}+x-2 n-2\right)\left(x^{2}-\right.$ $x-2 n-2),(n \geq 1)$.

(12) $P\left(S_{18}(n, p, q, t), x\right)=x^{2 n(t-1)}(x+1)^{p+q-2}(x-1)^{p+q-2}\left(x^{2}-t\right)^{2(n-1)}\left[x^{6}-4 x^{5}-(2 n+\right.$ $p+q+t-6) x^{4}+(6 n+2 p+2 q+4 t-4) x^{3}-(6 n+p-n p+q-n q-p q+6 t-p t-$ $\left.q t-1) x^{2}+(2 n-n p-n q+4 t-2 p t-2 q t) x-t+p t+q t-p q t\right]\left[x^{6}+4 x^{5}-(2 n+\right.$ $p+q+t-6) x^{4}-(6 n+2 p+2 q+4 t-4) x^{3}-(6 n+p-n p+q-n q-p q+6 t-$ $\left.p t-q t-1) x^{2}-(2 n-n p-n q+4 t-2 p t-2 q t) x-t+p t+q t-p q t\right],(n \geq 1, p \geq 0$, $q \geq 0, t \geq 0)$.

(12.1) $P\left(S_{18}(n, p, p, t), x\right)=x^{2 n(t-1)}(x+1)^{2 p-2}(x-1)^{2 p-2}\left(x^{2}-t\right)^{2(n-1)}\left[(x+1)^{2}-p\right][(x-$ $\left.1)^{2}-p\right]\left[x^{4}-2 x^{3}-(p+t+2 n-1) x^{2}+2(n+t) x+t(p-1)\right]\left[x^{4}+2 x^{3}-(p+t+2 n-\right.$ 1) $\left.x^{2}-2(n+t) x+t(p-1)\right],(n \geq 1, p \geq 0, t \geq 0)$. 
(12.2) $P\left(S_{18}(n, t, t, t), x\right)=x^{2 n(t-1)}(x+1)^{2 t-2}(x-1)^{2 t-2}\left(x^{2}-t\right)^{2(n-1)}\left[(x+1)^{2}-t\right]\left[(x-1)^{2}-\right.$ $t]\left[x^{4}-2 x^{3}-(2 t+2 n-1) x^{2}+2(n+t) x+t(t-1)\right]\left[x^{4}+2 x^{3}-(2 t+2 n-1) x^{2}-\right.$ $2(n+t) x+t(t-1)],(n \geq 1, t \geq 0)$.

(12.3) $P\left(S_{18}(n, 1,1,1), x\right)=x^{4}(x+2)(x+1)^{2 n-1}(x-1)^{2 n-1}(x-2)\left(x^{2}+x-2 n-2\right)\left(x^{2}-\right.$ $x-2 n-2),(n \geq 1)$.

(12.4) $P\left(S_{18}\left(2 k^{2}, k^{2}, k^{2}, k^{2}\right), x\right)=x^{4 k^{2}\left(k^{2}-1\right)}(x+k+1)(x+k)^{2\left(2 k^{2}-1\right)}(x+k-1)(x+1)^{2 k^{2}-2}(x-$ $1)^{2 k^{2}-2}(x-k+1)(x-k)^{2\left(2 k^{2}-1\right)}(x-k-1)\left[x^{2}+(2 k+1) x-k(k-1)\right]\left[x^{2}-(2 k+\right.$ $1) x-k(k-1)]\left[x^{2}+(2 k-1) x-k(k+1)\right]\left[x^{2}-(2 k-1) x-k(k+1)\right],(k \geq 1)$.

(13) $P\left(S_{19}(m, n, p, t), x\right)=x^{m n(t-1)+n}(x+1)^{n(p-1)}(x-1)^{n(p-1)}\left[x^{4}-(m+t+p+1) x^{2}+\right.$ $m+t+p t]^{n-1}\left(x^{2}-t\right)^{n(m-1)}\left\{x^{6}-(m+n+m n+p+n p+t+1) x^{4}+[m+n+m n+\right.$ $\left.\left.m n^{2}-2 n p+2 m n p+m n^{2} p+n p^{2}+t(1+n+p+n p)\right] x^{2}-n(p-1)^{2}(m n+t)\right\},(m \geq 1$, $n \geq 1, p \geq 1, t \geq 0)$.

(13.1) $P\left(S_{19}(m, n, 1, t), x\right)=x^{m n(t-1)+n+2}\left(x^{2}-t\right)^{n(m-1)}\left[x^{4}-(m+t+2) x^{2}+m+2 t\right]^{n-1}\left[x^{4}-\right.$ $(m+2 n+m n+t+2) x^{2}+(n+1)(m+2 m n+2 t),(m \geq 1, n \geq 1, t \geq 0)$.

(13.2) $P\left(S_{19}(2, n, 1,1), x\right)=x^{n+2}(x+1)^{2 n-1}(x-1)^{2 n-1}(x+2)^{n-1}(x-2)^{n-1}\left(x^{2}+x-2 n-\right.$ $2)\left(x^{2}-x-2 n-2\right),(n \geq 1)$.

(14) $P\left(S_{20}(n, p, q), x\right)=(x+1)^{p(n-1)+q}(x-1)^{p(n-1)+q}\left(x^{2}-x-n\right)^{p-1}\left(x^{2}+x-n\right)^{p-1}\left(x^{2}-\right.$ $x-p q-n)\left(x^{2}+x-p q-n\right),(n \geq 1, p \geq 1, q \geq 1)$.

(15) $P\left(S_{21}(m, t), x\right)=x^{2 m(t-1)+2}\left(x^{2}-x-t\right)^{m-1}\left(x^{2}+x-t\right)^{m-1}\left(x^{2}-x-m-t\right)\left(x^{2}+x-m-t\right)$, $(m \geq 1, t \geq 0)$.

Proof. We only prove (2) and (10). The characteristic polynomials of the other 13 types can be obtained similarly.

(2). By properly ordering the vertices of the graph $S_{2}(n, t)$, the adjacency matrix $A=A\left(S_{2}(n, t)\right)$ of $S_{2}(n, t)$ can be written as the $(n t+n+2) \times(n t+n+2)$ matrix such that

$$
A=A\left(S_{2}(n, t)\right)=\left[\begin{array}{llllll}
A_{11} & A_{12} & \ldots & A_{1 n} & B_{1} & 0_{t \times 2} \\
A_{21} & A_{22} & \ldots & A_{2 n} & B_{2} & 0_{t \times 2} \\
\vdots & \vdots & \ddots & \vdots & \vdots & \vdots \\
A_{n 1} & A_{n 2} & \ldots & A_{n n} & B_{n} & 0_{t \times 2} \\
B_{1}^{T} & B_{2}^{T} & \ldots & B_{n}^{T} & 0_{n \times n} & J_{n \times 2} \\
0_{2 \times t} & 0_{2 \times t} & \ldots & 0_{2 \times t} & J_{2 \times n} & 0_{2 \times 2}
\end{array}\right],
$$

where $A_{i j}=0_{t \times t}$ for $i=1,2, \ldots, n$ and $j=1,2, \ldots, n$, and

$$
B_{k}=\left[a_{i j}^{(k)}\right]=\left\{\begin{array}{ll}
1 & \text { if } j=k \\
0 & \text { otherwise }
\end{array}, \quad B_{k} \in R^{t \times n}, \text { for } k=1,2, \ldots, n .\right.
$$

Then we have 

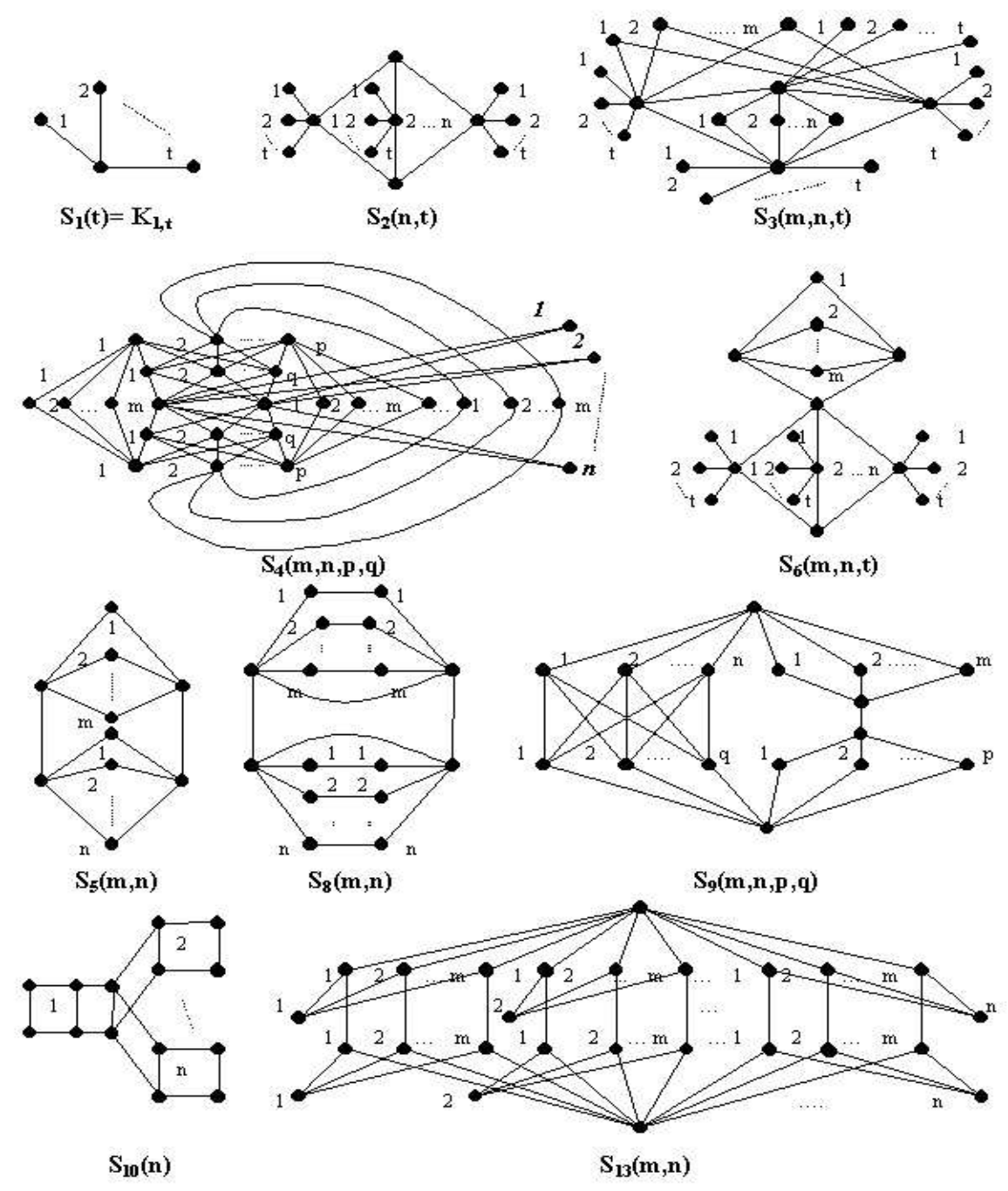

Figure 3: Nonregular bipartite graphs.

$$
P\left[S_{2}(n, t), x\right]=\left|x I_{n t+n+2}-A\left(S_{2}(n, t)\right)\right|=\left|\begin{array}{cccccc}
x I_{t} & 0_{t \times t} & \ldots & 0_{t \times t} & -B_{1} & 0_{t \times 2} \\
0_{t \times t} & x I_{t} & \ldots & 0_{t \times t} & -B_{2} & 0_{t \times 2} \\
\vdots & \vdots & \ddots & \vdots & \vdots & \vdots \\
0_{t \times t} & 0_{t \times t} & \ldots & x I_{t} & -B_{n} & 0_{t \times 2} \\
-B_{1}^{T} & -B_{2}^{T} & \ldots & -B_{n}^{T} & x I_{n} & -J_{n \times 2} \\
0_{2 \times t} & 0_{2 \times t} & \ldots & 0_{2 \times t} & -J_{2 \times n} & x I_{2}
\end{array}\right| .
$$

By careful calculation, we can prove that the characteristic polynomial of $S_{2}(n, t)$ is

$$
P\left(S_{2}(n, t), x\right)=x^{n(t-1)+2}\left(x^{2}-t\right)^{n-1}\left[x^{2}-(2 n+t)\right] .
$$

(10). By properly ordering the vertices of the graph $S_{13}(m, n)$, the adjacency matrix $A=A\left(S_{13}(m, n)\right)$ of $S_{13}(m, n)$ can be written as the $(2 m n+2 n+2) \times(2 m n+2 n+2)$ 

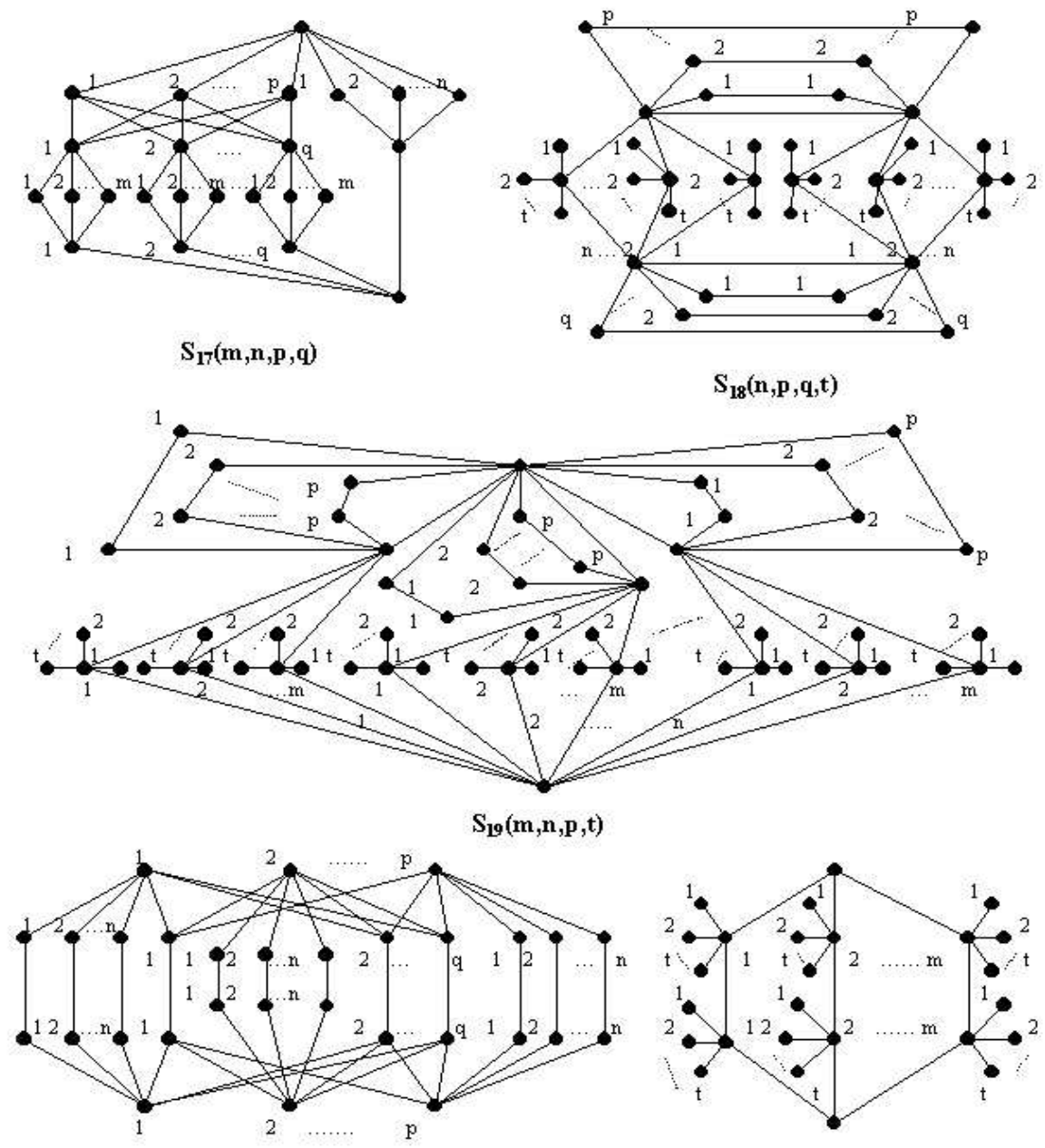

$S_{20}(n, p, q)$

$\mathrm{S}_{21}(\mathrm{~m}, \mathrm{t})$

Figure 4: Nonregular bipartite graphs.

matrix such that

$$
A=A\left(S_{13}(m, n)\right)=\left[\begin{array}{cc}
A_{0} & A_{1} \\
A_{1} & A_{0}
\end{array}\right]
$$

where

$$
A_{0}=\left[\begin{array}{cccccc}
0_{m \times m} & 0_{m \times m} & \ldots & 0_{m \times m} & B_{1} & J_{m \times 1} \\
0_{m \times m} & 0_{m \times m} & \ldots & 0_{m \times m} & B_{2} & J_{m \times 1} \\
\vdots & \vdots & \ddots & \vdots & \vdots & \vdots \\
0_{m \times m} & 0_{m \times m} & \ldots & 0_{m \times m} & B_{n} & J_{m \times 1} \\
B_{1}^{T} & B_{2}^{T} & \ldots & B_{n}^{T} & 0_{n \times n} & 0_{n \times 1} \\
J_{1 \times m} & J_{1 \times m} & \ldots & J_{1 \times m} & 0_{1 \times n} & 0
\end{array}\right]
$$




$$
A_{1}=\left[\begin{array}{cccccc}
I_{m} & 0_{m \times m} & \ldots & 0_{m \times m} & 0_{m \times n} & 0_{m \times 1} \\
0_{m \times m} & I_{m} & \ldots & 0_{m \times m} & 0_{m \times n} & 0_{m \times 1} \\
\vdots & \vdots & \ddots & \vdots & \vdots & \vdots \\
0_{m \times m} & 0_{m \times m} & \ldots & I_{m} & 0_{m \times n} & 0_{m \times 1} \\
0_{n \times m} & 0_{n \times m} & \ldots & 0_{n \times m} & 0_{n \times n} & 0_{n \times 1} \\
0_{1 \times m} & 0_{1 \times m} & \ldots & 0_{1 \times m} & 0_{1 \times n} & 0
\end{array}\right]
$$

and

$$
B_{k}=\left[a_{i j}^{(k)}\right]=\left\{\begin{array}{ll}
1 & \text { if } j=k \\
0 & \text { otherwise }
\end{array}, \quad B_{k} \in R^{m \times n}, \text { for } k=1,2, \ldots, n .\right.
$$

In view of Lemma 2.1, we distinguish between the following two cases.

Case 1. Let $b_{0}=\left|x I_{m n+n+1}-\left(A_{0}+A_{1}\right)\right|$. Then we have

$$
b_{0}=\left|\begin{array}{cccccc}
(x-1) I_{m} & 0_{m \times m} & \ldots & 0_{m \times m} & -B_{1} & -J_{m \times 1} \\
0_{m \times m} & (x-1) I_{m} & \ldots & 0_{m \times m} & -B_{2} & J_{m \times 1} \\
\vdots & \vdots & \ddots & \vdots & \vdots & \vdots \\
0_{m \times m} & 0_{m \times m} & \ldots & (x-1) I_{m} & -B_{n} & -J_{m \times 1} \\
-B_{1}^{T} & -B_{2}^{T} & \ldots & -B_{n}^{T} & x I_{n} & 0_{n \times 1} \\
-J_{1 \times m} & -J_{1 \times m} & \ldots & -J_{1 \times m} & 0_{1 \times n} & x
\end{array}\right|
$$

By careful calculation, we can find

$$
b_{0}=x(x-1)^{n(m-1)}\left(x^{2}-x-m\right)^{n-1}\left[x^{2}-x-m(n+1)\right] .
$$

Case 2. Let $b_{1}=\left|x I_{m n+n+1}-\left(A_{0}-A_{1}\right)\right|$. Then we have

$$
b_{1}=\left|\begin{array}{cccccc}
(x+1) I_{m} & 0_{m \times m} & \ldots & 0_{m \times m} & -B_{1} & -J_{m \times 1} \\
0_{m \times m} & (x+1) I_{m} & \ldots & 0_{m \times m} & -B_{2} & J_{m \times 1} \\
\vdots & \vdots & \ddots & \vdots & \vdots & \vdots \\
0_{m \times m} & 0_{m \times m} & \ldots & (x+1) I_{m} & -B_{n} & -J_{m \times 1} \\
-B_{1}^{T} & -B_{2}^{T} & \ldots & -B_{n}^{T} & x I_{n} & 0_{n \times 1} \\
-J_{1 \times m} & -J_{1 \times m} & \ldots & -J_{1 \times m} & 0_{1 \times n} & x
\end{array}\right|
$$

By careful calculation, we can find

$$
b_{1}=x(x+1)^{n(m-1)}\left(x^{2}+x-m\right)^{n-1}\left[x^{2}+x-m(n+1)\right] .
$$

Hence, the characteristic polynomial of $S_{13}(m, n)$ is

$$
\begin{aligned}
& P\left(S_{13}(m, n), x\right)=x^{2}(x+1)^{n(m-1)}(x-1)^{n(m-1)}\left(x^{2}+x-m\right)^{n-1}\left[x^{2}+x\right. \\
& -m(n+1)]\left(x^{2}-x-m\right)^{n-1}\left[x^{2}-x-m(n+1)\right] .
\end{aligned}
$$

The proof is now complete.

We note that these classes of graphs in Figures 3 and 4 are constructed from the smaller graphs in Figures 1 and 2 (or Figures 4 and 5 of [2]). We believe that it is useful to construct new classes of integral graphs by using this method. 


\section{Nonregular integral bipartite graphs}

In this section, by using number theory and computer search, we shall obtain some new classes of integral graphs from Theorem 3.2. All these classes are infinite and consist of connected graphs except for several disconnected graphs for which one or more of their parameters are taken zero.

Theorem 4.1. (see [5, 7]) The tree $K_{1, t}$ is integral if and only if $t$ is a perfect square.

Theorem 4.2. The graph $S_{2}(n, t)$ is integral if and only if one of the following holds: (i) $t$ and $2 n+t$ are perfect squares, or (ii) $n=1$ and $t+2$ is a perfect square, where $t(\geq 0)$ and $n(\geq 1)$ are integers.

In particular, we have the following results for the graph $S_{2}(n, t)$.

(1) If the graph $S_{2}(n, t)$ is integral, and $n(\geq 2), t(\geq 0)$ are integers, then for any positive integer $k$ the graph $S_{2}\left(n k^{2}, t k^{2}\right)$ is integral.

(2) If the graph $S_{2}(1, t-2)=K_{1, t}$ is integral, and $t$ is positive integer, then for any positive integer $k$ the graph $S_{2}\left(1, t k^{2}-2\right)=K_{1, t k^{2}}$ is integral.

(3) If $t=a^{2} \geq 0, n=\frac{b^{2}-a^{2}}{2} \geq 1, b>a$, and $a, b, n(\geq 1), t(\geq 0)$ are integers, then for any positive integer $k$ the graph $S_{2}\left(n k^{2}, t k^{2}\right)$ is integral.

Proof. By (2) of Theorem 3.2, we know

$P\left(S_{2}(n, t), x\right)=x^{n(t-1)+2}\left(x^{2}-t\right)^{n-1}\left[x^{2}-(2 n+t)\right],(n \geq 1, t \geq 0)$.

Hence, a sufficient and necessary condition for the graph $S_{2}(n, t)$ to be integral is the following: (i) when $n \geq 2, t$ and $2 n+t$ are perfect squares, or (ii) when $n=1, t+2$ is a perfect square, where $t(\geq 0)$ and $n(\geq 1)$ are integers.

By (2) of Theorem 3.2, we also get

$P\left(S_{2}\left(n k^{2}, t k^{2}\right), x\right)=x^{n k^{2}\left(t k^{2}-1\right)+2}\left(x^{2}-t k^{2}\right)^{n-1}\left[x^{2}-(2 n+t) k^{2}\right],(n \geq 1, t \geq 0, k \geq 1)$.

(1) Because the graph $S_{2}(n, t)$ is integral, and $n(\geq 2), t(\geq 0), k(\geq 1)$ are integers, we get that $t$ and $2 n+t$ are perfect squares. Then the graph $S_{2}\left(n k^{2}, t k^{2}\right)$ is integral.

(2) Because the graph $S_{2}(1, t-2)=K_{1, t}$ is integral, and $t, k$ are positive integers. Then $t$ must be a perfect square. Hence the graph $S_{2}\left(1, t k^{2}-2\right)=K_{1, t k^{2}}$ is integral.

(3) Because $t=a^{2} \geq 0, n=\frac{b^{2}-a^{2}}{2} \geq 1, b>a$, and $a, b, n(\geq 1), t(\geq 0), k(\geq 1)$ are integers, by (2) of Theorem 3.2, it follows

$$
P\left(S_{2}\left(\frac{b^{2}-a^{2}}{2}, a^{2}\right), x\right)=x^{\frac{b^{2}-a^{2}}{2}\left(a^{2}-1\right)+2}\left(x^{2}-a^{2}\right)^{\frac{b^{2}-a^{2}}{2}-1}\left(x^{2}-b^{2}\right) .
$$

So, the graph $S_{2}(n, t)=S_{2}\left(\frac{b^{2}-a^{2}}{2}, a^{2}\right)$ is integral. By Theorem 4.2 or Theorem $3.2(2)$, also the graph $S_{2}\left(n k^{2}, t k^{2}\right)=S_{2}\left(\frac{b^{2}-a^{2}}{2} \cdot k^{2}, a^{2} k^{2}\right)$ is integral.

Theorem 4.3. The graph $S_{3}(m, n, t)(n \geq m \geq 1, t \geq 0)$ is integral if and only if one of the following holds:

(1) For $m=n$, $t$ is a perfect square, and $2 n+t=k(k+2)$, where $k$ is a positive integer. 
(2) For $m<n$, let $(2 m+t, 2 n+t)=d$, $d$ is a positive integer but not a perfect square, and $m, n, t$ are given via

$$
2 m+t=d\left(\frac{y_{k}-y_{l}}{2}\right)^{2}, 2 n+t=d\left(\frac{y_{k}+y_{l}}{2}\right)^{2}, k>l>0, \text { and } t=t_{1}^{2}
$$

where $y_{k}, y_{l}$ are odd or even, $y_{k}, y_{l} \in\left\{y_{n} \mid y_{0}=0, y_{1}=b_{1}, y_{n+2}=a_{1} y_{n+1}-y_{n},(n \geq 0)\right\}$, $t_{1}$ is a nonnegative integer, and $a_{1}+b_{1} \sqrt{d}$ is the fundamental solution of Eq.(10).

(Examples are presented in Table 1. Table 1 is obtained by computer search, where a and $b$ be those of Eqs.(13) in Theorem 4.3, $1 \leq a \leq 15, a \leq b \leq a+10,1 \leq m<n$, $0 \leq t \leq 2500$.)

\begin{tabular}{|c|c|c|c|c||c|c|c|c|c||c|c|c|c|c|}
\hline$a$ & $b$ & $m$ & $n$ & $t$ & $a$ & $b$ & $m$ & $n$ & $t$ & $a$ & $b$ & $m$ & $n$ & $t$ \\
\hline 5 & 9 & 13 & 37 & 1 & 5 & 9 & 9 & 33 & 9 & 5 & 9 & 1 & 25 & 25 \\
\hline 14 & 20 & 100 & 196 & 0 & 14 & 20 & 98 & 194 & 4 & 14 & 20 & 92 & 188 & 16 \\
\hline 14 & 20 & 82 & 178 & 36 & 14 & 20 & 68 & 164 & 64 & 14 & 20 & 50 & 146 & 100 \\
\hline 14 & 20 & 28 & 124 & 144 & 14 & 20 & 2 & 98 & 196 & $/$ & $/$ & $/$ & $/$ & $/$ \\
\hline
\end{tabular}

Table 1: Integral graphs $S_{3}(m, n, t)=S_{3}(n, m, t)$.

Proof. By (3) of Theorem 3.2, we know that the graph $S_{3}(m, n, t)(n \geq m \geq 1, t \geq 0)$ is integral if and only if $t\left(=t_{1}^{2}\right)$ is a perfect square, and there exist nonnegative integers $a$ and $b$ such that $x^{4}-2(m+n+t+2) x^{2}+(2 m+t)(2 n+t)$ can be factorized as $\left(x^{2}-a^{2}\right)\left(x^{2}-b^{2}\right)$.

Next we discuss the following two cases:

Case 1. When $1 \leq m=n, t \geq 0$, by (3) of Theorem 3.2, we get

$P\left(S_{3}(n, n, t), x\right)=x^{2 n+4(t-1)}\left(x^{2}-t\right)^{2}\left(x^{2}+2 x-2 n-t\right)\left(x^{2}+2 x-2 n-t\right)$.

Hence, the graph $S_{3}(n, n, t)$ is integral if and only if $t$ is a perfect square, and $2 n+t=$ $k(k+2)$, where $n(\geq 1), k(\geq 1)$ and $t(\geq 0)$ are integers.

Case 2. When $1 \leq m<n, t \geq 0$, by (3) of Theorem 3.2, the necessary and sufficient condition for $S_{3}(m, n, t)$ to be an integral graph is that there are nonnegative integers $a$ and $b$ such that the following Diophantine equations (13) have solutions.

$$
\left\{\begin{array}{l}
t=t_{1}^{2}, \\
a^{2}+b^{2}=2 m+2 n+2 t+4, \\
a^{2} b^{2}=(2 m+t)(2 n+t),
\end{array}\right.
$$

Let $(2 m+t, 2 n+t)=d, 1 \leq m<n, a<b$. By (13), we find

$$
2 m+t=d m_{1}^{2}, \quad 2 n+t=d n_{1}^{2}, \quad a b=d m_{1} n_{1},
$$

where $m_{1}$ and $n_{1}$ are nonnegative integers, $m_{1}<n_{1}$, and $\left(m_{1}, n_{1}\right)=1$. By (13) and (14), we obtain

$$
(a+b)^{2}-d\left(m_{1}+n_{1}\right)^{2}=4 .
$$

We discuss the following two cases. 
Case 2.1 If $d$ is a perfect square, clearly, the Diophantine equation (15) has no integral solutions, then the graph $S_{3}(m, n, t)$ is not an integral graph.

Case 2.2 If $d$ is a positive integer but not a perfect square, then the equation (15) is a Pell equation. Let $\varepsilon_{1}=\frac{a_{1}+b_{1} \sqrt{d}}{2}$, where $a_{1}+b_{1} \sqrt{d}$ is the fundamental solution of the equation (10). From (15), we deduce that

$$
a+b=\varepsilon_{1}^{k}+{\overline{\varepsilon_{1}}}^{k}, \quad m_{1}+n_{1}=\frac{\varepsilon_{1}^{k}-{\overline{\varepsilon_{1}}}^{k}}{\sqrt{d}}, \quad k>0,
$$

where $\overline{\varepsilon_{1}}=\frac{a_{1}-b_{1} \sqrt{d}}{2}$ and $\varepsilon_{1} \overline{\varepsilon_{1}}=1$ (see Lemma 2.8).

By using (16) and $a b=d m_{1} n_{1}$ (see (14)), we get

$$
\left(2 b-\left(\varepsilon_{1}^{k}+{\overline{\varepsilon_{1}}}^{k}\right)\right)^{2}-d\left(2 n_{1}-\frac{\varepsilon_{1}^{k}-{\overline{\varepsilon_{1}}}^{k}}{\sqrt{d}}\right)^{2}=4 .
$$

Thus, we have $\quad 2 b=\varepsilon_{1}^{k}+\bar{\varepsilon}_{1}^{k}+\varepsilon_{1}^{l}+{\overline{\varepsilon_{1}}}^{l}, \quad 2 n_{1}=\frac{\varepsilon_{1}^{k}-{\overline{\varepsilon_{1}}}^{k}}{\sqrt{d}}+\frac{\varepsilon_{1}^{l}-{\overline{\varepsilon_{1}}}^{l}}{\sqrt{d}}, \quad l>0$.

Hence, $\quad m_{1}=\frac{\varepsilon_{1}^{k}-{\overline{\varepsilon_{1}}}^{k}}{2 \sqrt{d}}-\frac{\varepsilon_{1}^{l}-{\overline{\varepsilon_{1}}}^{l}}{2 \sqrt{d}}, \quad n_{1}=\frac{\varepsilon_{1}^{k}-{\overline{\varepsilon_{1}}}^{k}}{2 \sqrt{d}}+\frac{\varepsilon_{1}^{l}-\bar{\varepsilon}_{1}^{l}}{2 \sqrt{d}}, \quad k>l>0$.

Let

$$
y_{n}=\frac{\varepsilon_{1}^{n}-{\overline{\varepsilon_{1}}}^{n}}{\sqrt{d}}, \quad n=0,1,2, \ldots
$$

Then we get the Pell sequence

$$
y_{0}=0, \quad y_{1}=b_{1}, \quad y_{n+2}=a_{1} y_{n+1}-y_{n}, \quad(n \geq 0) .
$$

Hence, all integral graphs $S_{3}(m, n, t)$ (where $\left.1 \leq m<n\right)$ are given via

$$
2 m+t=d\left(\frac{y_{k}-y_{l}}{2}\right)^{2}, 2 n+t=d\left(\frac{y_{k}+y_{l}}{2}\right)^{2}, k>l>0, \text { and } t=t_{1}^{2},
$$

where $t_{1}$ is a nonnegative integer.

The proof is now complete.

Corollary 4.4. For $m=n$ the graph $S_{3}(n, n, t)$ is integral if and only if one of the following holds: (i) $m=n, t=n^{2}$, (ii) $m=n=2 l(l+1)-2 k^{2} \geq 1, t=4 k^{2} \geq 0$, or (iii) $m=n=2 l(l+2)+1-2 k(k+1) \geq 1, t=(2 k+1)^{2}$, where $m=n(\geq 1), t(\geq 0), l$ $(\geq 0), k(\geq 0)$ are integers.

Proof. Because $m=n$, by Theorem 4.3 (1), we know that the graph $S_{3}(n, n, t)$ is integral if and only if $t\left(=k_{1}^{2}\right)$ is a perfect square, and $2 n+t=s(s+2)$, where $n(\geq 1), s(\geq 1)$ and $t(\geq 0)$ are integers. Thus $n=\frac{1}{2}\left(s(s+2)-k_{1}^{2}\right)$ must be a positive integer. Hence we discuss the following three cases:

(i). If $k_{1}=n$, then $m=n, t=n^{2}, 2 n+t=n(n+2)$.

(ii). If $k_{1}$ is even, then $s$ must be even. So, let $k_{1}=2 k$ and $s=2 l$, where $k$ and $l$ are positive integers. Hence $m=n=2 l(l+1)-2 k^{2} \geq 1, t=4 k^{2} \geq 0$.

(iii). If $k_{1}$ is odd, then $s$ must be odd. So, let $k_{1}=2 k+1$ and $s=2(l+1)$, where $k$ and $l$ are positive integers. Hence $m=n=2 l(l+2)+1-2 k(k+1) \geq 1, t=(2 k+1)^{2}$. 
Theorem 4.5. The graph $S_{4}(m, n, p, q)$ is integral if and only if there exist nonnegative integers $a$ and $b$ such that $x^{4}-(2 m+2 n+4 q+p q) x^{2}+4 m n+8 m q+2 n p q$ can be factorized as $\left(x^{2}-a^{2}\right)\left(x^{2}-b^{2}\right)$, and one of the following holds: (i) $2 m$ and $p q\left(=c^{2}\right)$ are perfect squares, (ii) $p=1, q\left(=c^{2}\right)$ is a perfect square, where $m(\geq 0), n(\geq 0) p(\geq 1)$ and $q$ $(\geq 1)$ are integers.

In particular, let $a, b, c, m, n, p, q$ be as in Theorem 4.5, and let $m(\geq 1), n(\geq 1)$, $p(\geq 1), q(\geq 1), l(\geq 1), k(\geq 1), r(\geq 1), b_{1}, a, b, c$ be integers. Then we have the following results for the graph $S_{4}(m, n, p, q)$.

(1) If $m=n$, then the graph $S_{4}(n, n, p, q)$ is integral if and only if $2 n, p q$ and $2 n+4 q+p q$ are perfect squares, where $n(\geq 0), p(\geq 1)$ and $q(\geq 1)$ are integers.

(2) If $m=n, p=q$, then the graph $S_{4}(n, n, p, p)$ is integral if and only if $2 n$ and $2 n+p^{2}+4 p$ are perfect squares, where $n$ and $p$ are positive integers.

(3) If $m=n=2, p=q$, then the graph $S_{4}(m, n, p, q)$ is integral.

(4) If $m=n=2 l^{2} r^{2}, q=p k^{2} r^{2}, a=2 l r, b=b_{1} r, c=p k r$, and let $b_{1}, p, k, l$ be positive integers satisfying the Diophantine equation

$$
b_{1}^{2}-\left(p^{2}+4 p\right) k^{2}=4 l^{2} .
$$

Then the graph $S_{4}(m, n, p, q)$ is integral.

(5) If $m=n=2 l^{2} r^{2}, p=q k^{2} r^{2}, a=2 l r, b=b_{1} r, c=q k r$, and let $b_{1}, q, k, l$ be positive integers satisfying the Diophantine equation

$$
b_{1}^{2}-\left(q^{2}+4 q\right) k^{2}=4 l^{2} .
$$

Then the graph $S_{4}(m, n, p, q)$ is integral.

(6) If $m=n=2 l^{2} r^{2}, p=p_{1}^{2}, q=q_{1}^{2} r^{2}, a=2 l r, b=b_{1} r, c=p_{1} q_{1} r$, and let $b_{1}, p_{1}, q_{1}, l$ be positive integers satisfying the Diophantine equation

$$
b_{1}^{2}-\left(p_{1}^{2}+4\right) q_{1}^{2}=4 l^{2} .
$$

Then the graph $S_{4}(m, n, p, q)$ is integral.

(7) If $m=n=2 l^{2}, p=q, a=2 l, c=p$, and let $b, p, l$ be positive integers satisfying the Diophantine equation

$$
b^{2}-4 l^{2}=p(p+4) .
$$

Then the graph $S_{4}(m, n, p, q)$ is integral.

(8) If $m=n$, let $a, b, c, m, n, p$ and $q$ be given as in Table 2. Then the graph $S_{4}(m, n, p, q)$ is integral. (Table 2 is obtained by computer search, where $0 \leq a \leq 10$, $a \leq b \leq a+10, m=n$ and $m, n, p$ and $q$ are not as in (3), but represent solutions of (4)-(7).) 
(9) If $m \neq n, p=1$, and (i) $a=4, b=16, c=6, m=2, n=44, p=1$ and $q=36$, or (ii) $a=6, b=19, c=3, m=14, n=162, p=1, q=9$. Then the graph $S_{4}(m, n, p, q)$ is integral.(Here $a, b, c, m, n, p$ and $q$ are obtained by computer search, and $0 \leq a \leq 15, a \leq b \leq a+30, m \neq n, p=1)$.

(10) If $m \neq n$, and $a, b, c, m, n, p, q$ are given in Table 3. Then the graph $S_{4}(m, n, p, q)$ is integral. (Table 3 is obtained by computer search, where $1 \leq a \leq 10, a \leq b \leq a+20$ and $m \neq n$.)

(11) If $m=0$ or $n=0$, and $a, b, c, m, n, p, q$ given in Table 4 , then the graph $S_{4}(m, n, p, q)$ is integral.(Table 4 is obtained by computer search, where $1 \leq a \leq 10$, $a \leq b \leq a+30$ and $m=0$ or $n=0$.)

\begin{tabular}{|c|c|c|c|c|c|c||c|c|c|c|c|c|c|}
\hline$a$ & $b$ & $c$ & $m$ & $n$ & $p$ & $q$ & $a$ & $b$ & $c$ & $m$ & $n$ & $p$ & $q$ \\
\hline 2 & 7 & 3 & 2 & 2 & 1 & 9 & 4 & 6 & 2 & 8 & 8 & 1 & 4 \\
\hline 4 & 6 & 4 & 8 & 8 & 16 & 1 & 4 & 8 & 4 & 8 & 8 & 2 & 8 \\
\hline 4 & 8 & 6 & 8 & 8 & 12 & 3 & 4 & 10 & 6 & 8 & 8 & 3 & 12 \\
\hline 4 & 12 & 8 & 8 & 8 & 4 & 16 & 4 & 14 & 6 & 8 & 8 & 1 & 36 \\
\hline 4 & 14 & 10 & 8 & 8 & 5 & 20 & 4 & 14 & 12 & 8 & 8 & 16 & 9 \\
\hline 6 & 7 & 3 & 18 & 18 & 9 & 1 & 6 & 9 & 3 & 18 & 18 & 1 & 9 \\
\hline 6 & 9 & 5 & 18 & 18 & 5 & 5 & 6 & 11 & 9 & 18 & 18 & 81 & 1 \\
\hline 6 & 12 & 6 & 18 & 18 & 2 & 18 & 6 & 12 & 10 & 18 & 18 & 50 & 2 \\
\hline 6 & 14 & 12 & 18 & 18 & 36 & 4 & 6 & 15 & 9 & 18 & 18 & 3 & 27 \\
\hline 8 & 12 & 4 & 32 & 32 & 1 & 16 & 8 & 12 & 8 & 32 & 32 & 16 & 4 \\
\hline 8 & 16 & 8 & 32 & 32 & 2 & 32 & 8 & 16 & 12 & 32 & 32 & 12 & 12 \\
\hline 8 & 18 & 16 & 32 & 32 & 256 & 1 & 10 & 11 & 3 & 50 & 50 & 3 & 3 \\
\hline 10 & 12 & 6 & 50 & 50 & 18 & 2 & 10 & 14 & 8 & 50 & 50 & 8 & 8 \\
\hline 10 & 15 & 5 & 50 & 50 & 1 & 25 & 10 & 15 & 11 & 50 & 50 & 121 & 1 \\
\hline 10 & 16 & 12 & 50 & 50 & 48 & 3 & 10 & 17 & 9 & 50 & 50 & 3 & 27 \\
\hline 10 & 18 & 14 & 50 & 50 & 28 & 7 & 10 & 19 & 15 & 50 & 50 & 25 & 9 \\
\hline 10 & 20 & 10 & 50 & 50 & 2 & 50 & $/$ & $/$ & $/$ & $/$ & $/$ & $/$ & $/$ \\
\hline
\end{tabular}

Table 2: Integral graphs $S_{4}(m, n, p, q)$.

Proof. By using (4), (4.1) and (4.2) of Theorem 3.2, this theorem and (1), (2) of Theorem 4.5 are shown similarly to Theorem 4.2 .

(3) Because $m=n=2, p=q$, we have by (4.3) of Theorem 3.2,

$P\left(S_{4}(2,2, p, p), x\right)=x^{4 p}(x+p+2)(x+p)(x+2)^{p}(x-2)^{p}(x-p)(x-p-2)$,

where $p$ is a positive integer. Hence, the graph $S_{4}(2,2, p, p)$ is integral.

(4)-(7) When $m=n$, by Theorem 4.5, the graph $S_{4}(m, n, p, q)$ is integral if and only if $2 n$ and $p q\left(=c^{2}\right)$ are perfect squares, and $x^{4}-(2 m+2 n+4 q+p q) x^{2}+4 m n+8 m q+2 n p q=$ $\left(x^{2}-2 n\right)\left[x^{2}-(2 n+4 q+p q)\right]$ can be factorized as $\left(x^{2}-a^{2}\right)\left(x^{2}-b^{2}\right)$, where $m=n(\geq 1)$, 


\begin{tabular}{|c|c|c|c|c|c|c||c|c|c|c|c|c|c|}
\hline$a$ & $b$ & $c$ & $m$ & $n$ & $p$ & $q$ & $a$ & $b$ & $c$ & $m$ & $n$ & $p$ & $q$ \\
\hline 4 & 6 & 4 & 2 & 14 & 16 & 1 & 4 & 8 & 6 & 2 & 12 & 9 & 4 \\
\hline 4 & 16 & 6 & 2 & 44 & 1 & 36 & 6 & 10 & 8 & 2 & 26 & 16 & 4 \\
\hline 6 & 14 & 8 & 2 & 50 & 4 & 16 & 6 & 14 & 8 & 50 & 2 & 4 & 16 \\
\hline 6 & 16 & 6 & 2 & 114 & 6 & 6 & 6 & 18 & 8 & 2 & 82 & 2 & 32 \\
\hline 6 & 22 & 12 & 162 & 2 & 12 & 12 & 7 & 24 & 15 & 162 & 8 & 15 & 15 \\
\hline 8 & 12 & 10 & 2 & 44 & 25 & 4 & 8 & 12 & 8 & 8 & 56 & 16 & 4 \\
\hline 8 & 12 & 6 & 18 & 60 & 9 & 4 & 8 & 12 & 6 & 50 & 28 & 9 & 4 \\
\hline 8 & 14 & 8 & 2 & 92 & 32 & 2 & 8 & 14 & 12 & 8 & 38 & 24 & 6 \\
\hline 8 & 16 & 12 & 8 & 48 & 9 & 16 & 8 & 18 & 8 & 2 & 152 & 16 & 4 \\
\hline 8 & 18 & 8 & 128 & 26 & 16 & 4 & 9 & 12 & 9 & 18 & 48 & 27 & 3 \\
\hline 10 & 14 & 12 & 2 & 66 & 36 & 4 & 10 & 16 & 12 & 2 & 86 & 16 & 9 \\
\hline 10 & 16 & 12 & 32 & 56 & 16 & 9 & 10 & 20 & 12 & 32 & 74 & 4 & 36 \\
\hline 10 & 30 & 24 & 18 & 66 & 9 & 64 & $/$ & $/$ & $/$ & $/$ & $/$ & $/$ & $/$ \\
\hline
\end{tabular}

Table 3: Integral graphs $S_{4}(m, n, p, q)$.

\begin{tabular}{|c|c|c|c|c|c|c||c|c|c|c|c|c|c|}
\hline$a$ & $b$ & $c$ & $m$ & $n$ & $p$ & $q$ & $a$ & $b$ & $c$ & $m$ & $n$ & $p$ & $q$ \\
\hline 1 & 4 & 3 & 2 & 0 & 9 & 1 & 2 & 8 & 6 & 8 & 0 & 9 & 4 \\
\hline 2 & 28 & 24 & 98 & 0 & 144 & 4 & 3 & 12 & 9 & 18 & 0 & 9 & 9 \\
\hline 4 & 16 & 12 & 32 & 0 & 9 & 1 & 4 & 21 & 15 & 98 & 0 & 25 & 9 \\
\hline 5 & 20 & 15 & 50 & 0 & 9 & 25 & 6 & 20 & 12 & 0 & 50 & 3 & 48 \\
\hline 6 & 24 & 18 & 72 & 0 & 9 & 36 & 7 & 28 & 21 & 98 & 0 & 9 & 49 \\
\hline 8 & 30 & 24 & 0 & 50 & 8 & 72 & 8 & 32 & 24 & 128 & 0 & 9 & 64 \\
\hline 9 & 36 & 27 & 162 & 0 & 9 & 81 & 10 & 40 & 30 & 200 & 0 & 9 & 100 \\
\hline
\end{tabular}

Table 4: Integral graphs $S_{4}(m, n, p, q)$.

$p(\geq 1), q(\geq 1), a, b$ and $c$ are integers. Without loss of generality, assume that $a^{2}=2 n$, $b^{2}=2 n+4 q+p q$. Hence, the graph $S_{4}(m, n, p, q)$ is integral if and only if the equations

$$
\left\{\begin{array}{l}
a^{2}=2 n, \\
b^{2}=2 n+4 q+p q, \\
p q=c^{2}
\end{array}\right.
$$

have only integral roots. We distinguish between the following four cases:

Case 1. Suppose that $m=n=2 l^{2} r^{2}, q=p k^{2} r^{2}, a=2 l r, b=b_{1} r, c=p k r$, where $l$ $(\geq 1), r(\geq 1), b_{1}$ and $k(\geq 1)$ are integers. By Eqs.(21), we get the Diophantine equation (17). From Theorem 4.5, we see that (4) of Theorem 4.5 is true.

Case 2. Suppose that $m=n=2 l^{2} r^{2}, p=q k^{2} r^{2}, a=2 l r, b=b_{1} r, c=q k r$, where $l$ $(\geq 1), r(\geq 1), b_{1}$ and $k(\geq 1)$ are integers. By Eqs.(21), we get the Diophantine equation (18). From Theorem 4.5, the result in (5) follows.

Case 3. Suppose that $m=n=2 l^{2} r^{2}, p=p_{1}^{2}, a=2 l r, b=b_{1} r, c=p_{1} q_{1} r$ and $q=q_{1}^{2} r^{2}$, where $l(\geq 1), r(\geq 1), b_{1}, p_{1}(\geq 1)$ and $q_{1}(\geq 1)$ are integers. Eqs.(21) yields the 
Diophantine equation (19), which proves Theorem 4.5 (6).

Case 4. Suppose that $m=n=2 l^{2}, q=p, a=2 l$ and $c=p$, where $l(\geq 1)$ and $p(\geq 1)$ are integers. Eqs.(21) leads to the Diophantine equation (20). This shows Theorem 4.5 (7).

The results in (8)-(11) can be shown similarly to (3) by using Theorem 3.2 (4).

Remark 4.6. For the Diophantine equations (17)-(19) and (20), all positive integral solutions can be found from Lemmas 2.2-2.8 and Lemma 2.9, respectively. This shows that there are infinitely many integral graphs $S_{4}(m, n, p, q)$.

Theorem 4.7. The graph $S_{5}(m, n)(m \leq n)$ is integral if and only if one of the following holds:

(1) $m=n=\frac{1}{2} k(k+1)$, where $k(\geq 0)$ is an integer.

(2) $m=0, n=2 k(k+1)$, where $k(\geq 0)$ is an integer.

(3) $m<n$, let $(m, n)=d, 2 d$ is a positive integer but not a perfect square, and $m$, $n$ are given by

$$
m=2 d\left(\frac{y_{k}-y_{l}}{2}\right)^{2}, \quad n=2 d\left(\frac{y_{k}+y_{l}}{2}\right)^{2}, \quad k>l>0,
$$

where $y_{k}, y_{l}$ are odd or even, $y_{k}, y_{l} \in\left\{y_{n} \mid y_{0}=0, y_{1}=b_{1}, y_{n+2}=2 a_{1} y_{n+1}-y_{n},(n \geq 0)\right\}$, and $a_{1}+b_{1} \sqrt{2 d}$ is the fundamental solution of the Diophantine equation

$$
x^{2}-2 d y^{2}=1
$$

Examples are presented in Table 5. (Table 5 is obtained by computer search, where a and $b$ are those of Eqs.(23), $1 \leq a \leq 155, a \leq b \leq a+80$ and $1 \leq m<n$.)

\begin{tabular}{|c|c|c|c||c|c|c|c||c|c|c|c|}
\hline$a$ & $b$ & $m$ & $n$ & $a$ & $b$ & $m$ & $n$ & $a$ & $b$ & $m$ & $n$ \\
\hline 7 & 10 & 25 & 49 & 22 & 27 & 243 & 363 & 41 & 58 & 841 & 1681 \\
\hline 76 & 85 & 2890 & 3610 & 115 & 126 & 6615 & 7935 & $/$ & $/$ & $/$ & $/$ \\
\hline
\end{tabular}

Table 5: Integral graphs $S_{5}(m, n)$.

Proof. (1)-(2) As in the proof of Theorem 4.3 (1), the results follow by using (5.1) and (5.2) of Theorem 3.2.

(3) By Theorem Theorem $3.2(5)$, the necessary and sufficient condition for $S_{5}(m, n)$ to be an integral graph is that there are positive integers $a$ and $b$ such that

$$
\left\{\begin{aligned}
a^{2}+b^{2} & =2 m+2 n+1 \\
a^{2} b^{2} & =4 m n
\end{aligned}\right.
$$


Let $(m, n)=d, 1 \leq m<n$. By (23), we find

$$
m=d m_{1}^{2}, \quad n=d n_{1}^{2}, \quad a b=2 d m_{1} n_{1},
$$

where $m_{1}$ and $n_{1}$ are positive integers, and $\left(m_{1}, n_{1}\right)=1$. By $(23)$ and $(24)$, we obtain

$$
(a+b)^{2}-2 d\left(m_{1}+n_{1}\right)^{2}=1 \text {. }
$$

We discuss the following two cases.

Case 1. If $2 d$ is a perfect square, clearly, the Diophantine equation (25) has no integral solutions. Then the graph $S_{5}(m, n)$ is not an integral graph.

Case 2. If $2 d$ is a positive integer but not a perfect square. Then Eq.(25) is a Pell equation. Let $\varepsilon_{1}=a_{1}+b_{1} \sqrt{2 d}$ be the fundamental solution of Eq.(22). From (25), we deduce that

$$
a+b=\frac{\varepsilon^{k}+\bar{\varepsilon}^{k}}{2}, \quad m_{1}+n_{1}=\frac{\varepsilon^{k}-\bar{\varepsilon}^{k}}{2 \sqrt{2 d}}, \quad k>0,
$$

where $\bar{\varepsilon}=a_{1}-b_{1} \sqrt{2 d}$ and $\varepsilon \bar{\varepsilon}=1$ (see Lemma 2.5).

By using (26) and $a b=2 d m_{1} n_{1}$ (see (24)), we get

$$
\left(2 b-\frac{\varepsilon^{k}+\bar{\varepsilon}^{k}}{2}\right)^{2}-2 d\left(2 n_{1}-\frac{\varepsilon^{k}-\bar{\varepsilon}^{k}}{2 \sqrt{2 d}}\right)^{2}=1 .
$$

Thus, we have $\quad 2 b=\frac{\varepsilon^{k}+\bar{\varepsilon}^{k}}{2}+\frac{\varepsilon^{l}+\bar{\varepsilon}^{l}}{2}, \quad 2 n_{1}=\frac{\varepsilon^{k}-\bar{\varepsilon}^{k}}{2 \sqrt{2 d}}+\frac{\varepsilon^{l}-\bar{\varepsilon}^{l}}{2 \sqrt{2 d}}, \quad l>0$.

Hence, $\quad m_{1}=\left(\frac{\varepsilon^{k}-\bar{\varepsilon}^{k}}{2 \sqrt{2 d}}-\frac{\varepsilon^{l}-\bar{\varepsilon}^{l}}{2 \sqrt{2 d}}\right) / 2, \quad n_{1}=\left(\frac{\varepsilon^{k}-\bar{\varepsilon}^{k}}{2 \sqrt{2 d}}+\frac{\varepsilon^{l}-\bar{\varepsilon}^{l}}{2 \sqrt{2 d}}\right) / 2, \quad k>l>0$.

$$
\text { Let } \quad y_{n}=\frac{\varepsilon^{n}-\bar{\varepsilon}^{n}}{2 \sqrt{2 d}}, \quad n=0,1,2, \ldots
$$

Then we get the Pell sequence (see [3])

$$
y_{0}=0, \quad y_{1}=b_{1}, \quad y_{n+2}=2 a_{1} y_{n+1}-y_{n}, \quad(n \geq 0) .
$$

Hence, all integral graphs $S_{5}(m, n)$ (where $1 \leq m<n$ ) are given by

$$
m=2 d\left(\frac{y_{k}-y_{l}}{2}\right)^{2}, \quad n=2 d\left(\frac{y_{k}+y_{l}}{2}\right)^{2}, \quad k>l>0 .
$$

The proof is now complete.

In a similar way the next results can be derived by using Theorem $3.2(6)$.

Theorem 4.8. The graph $S_{6}(m, n, t)$ is integral if and only if there exist nonnegative integers $a$ and $b$ such that $x^{4}-(2 m+2 n+t+2) x^{2}+2 n(2 m+1)+2 t(m+1)$ can be factorized as $\left(x^{2}-a^{2}\right)\left(x^{2}-b^{2}\right)$, and one of the following two conditions holds: (i) $t$ is a perfect square, (ii) $n=1$, where $m(\geq 0), n(\geq 1), t(\geq 0)$ or $m(\geq 0), n=t=0$.

In particular, we have the following results for the graph $S_{6}(m, n, t)$. 
(1) For $n=t=0$ the graph $S_{6}(m, 0,0)=K_{2, m+1} \cup K_{1}$ is integral if and only if $m=$ $2 k^{2}-1$, where $k$ is a positive integer.

(2) For $m=0, t=1$ the graph $S_{6}(0, n, 1)$ is integral if and only if $n=2 k^{2}-1$, where $k$ is a positive integer.

(3) For $t=0$, let $(2 m+1,2 n)=d$. We have the following results.

(i) If $d$ is a perfect square, then $S_{6}(m, n, 0)$ is not an integral graph.

(ii) If $d$ is a positive integer but not a perfect square, then all integral graphs $S_{6}(m, n, 0)$ (where $\left.1 \leq m<n\right)$ are given via

$$
2 m+1=d\left(\frac{y_{k}-y_{l}}{2}\right)^{2}, \quad 2 n=d\left(\frac{y_{k}+y_{l}}{2}\right)^{2}, \quad k>l>0,
$$

where $y_{k}, y_{l}$ are odd or even, $y_{k}, y_{l} \in\left\{y_{n} \mid y_{0}=0, y_{1}=b_{1}, y_{n+2}=2 a_{1} y_{n+1}-y_{n},(n \geq\right.$ $0)\}$, and $a_{1}+b_{1} \sqrt{d}$ is the fundamental solution of the Pell equation (2).

(Examples are presented in Table 6. The first solution in Table 6 is obtained by computer search, where $a$ and $b$ are those of Theorem 4.8, $0 \leq a \leq 100, a \leq b \leq$ $a+30$ and $m \geq 0, n \geq 0$.)

(4) For $n=1$, let $a, b, m, n, t$ be as in Theorem 4.8, and $a=1, b=2, m=0$ and $n=t=1$. Then the graph $S_{6}(0,1,1)$ is integral. (This solution is obtained by computer search, where $0 \leq a \leq 25, a \leq b \leq a+20, m \geq 0, t \geq 0$.)

(5) For $m=n-1, t=1$ the graph $S_{6}(n-1, n, 1)$ is integral if and only if $n=\frac{1}{2} k(k+1)$, where $k$ is a positive integer.

(6) For $m=n+1, t=1$ the graph $S_{6}(n+1, n, 1)$ is integral if and only if $n=$ $\frac{1}{2} k(k+1)-1$, where $k$ is a positive integer.

(7) For $m=n+1, t=9$, then the graph $S_{6}(n+1, n, 9)$ is integral if and only if $n=\frac{1}{2} k(k+1)-3$, where $k(\geq 2)$ is a positive integer.

(8) Let $a, b, m, n$ and $t$ be as in Theorem 4.8, and given in Table 7, then the graph $S_{6}(m, n, t)$ is integral. (Table 7 is obtained by computer search, where $1 \leq a \leq 8$, $a \leq b \leq a+20$ ). (Note that $m, n$, $t$ are different from those in Theorem 4.8(1)-(7).)

\begin{tabular}{|c|c|c|c|c||c|c|c|c|c|}
\hline$a$ & $b$ & $m$ & $n$ & $t$ & $a$ & $b$ & $m$ & $n$ & $t$ \\
\hline 12 & 14 & 73 & 96 & 0 & 2520 & 2522 & 3175537 & 3179904 & 0 \\
\hline
\end{tabular}

Table 6: Integral graphs $S_{6}(m, n, t)$, where $1 \leq m<n, t=0$. 


\begin{tabular}{|c|c|c|c|c||c|c|c|c|c||c|c|c|c|c|}
\hline$a$ & $b$ & $m$ & $n$ & $t$ & $a$ & $b$ & $m$ & $n$ & $t$ & $a$ & $b$ & $m$ & $n$ & $t$ \\
\hline 3 & 6 & 4 & 13 & 9 & 3 & 8 & 4 & 27 & 9 & 3 & 10 & 4 & 45 & 9 \\
\hline 3 & 12 & 4 & 67 & 9 & 3 & 14 & 4 & 93 & 9 & 3 & 16 & 4 & 123 & 9 \\
\hline 3 & 18 & 4 & 157 & 9 & 3 & 20 & 4 & 195 & 9 & 3 & 22 & 4 & 237 & 9 \\
\hline 4 & 5 & 11 & 4 & 9 & 5 & 6 & 12 & 5 & 25 & 5 & 6 & 16 & 9 & 9 \\
\hline 5 & 8 & 12 & 19 & 25 & 5 & 10 & 12 & 37 & 25 & 5 & 12 & 12 & 59 & 25 \\
\hline 5 & 14 & 12 & 85 & 25 & 5 & 16 & 12 & 115 & 25 & 5 & 18 & 12 & 149 & 25 \\
\hline 5 & 20 & 12 & 187 & 25 & 5 & 22 & 12 & 229 & 25 & 5 & 24 & 12 & 275 & 25 \\
\hline 6 & 7 & 18 & 11 & 25 & 6 & 7 & 22 & 15 & 9 & 6 & 7 & 23 & 6 & 25 \\
\hline 7 & 8 & 24 & 7 & 49 & 7 & 8 & 25 & 18 & 25 & 7 & 8 & 29 & 22 & 9 \\
\hline 7 & 8 & 30 & 13 & 25 & 7 & 10 & 24 & 25 & 49 & 7 & 12 & 24 & 47 & 49 \\
\hline 7 & 14 & 24 & 73 & 49 & 7 & 16 & 24 & 103 & 49 & 7 & 18 & 24 & 137 & 49 \\
\hline 7 & 20 & 24 & 175 & 49 & 7 & 22 & 24 & 217 & 49 & 7 & 24 & 24 & 263 & 49 \\
\hline 7 & 26 & 24 & 313 & 49 & 8 & 9 & 32 & 15 & 49 & 8 & 9 & 33 & 26 & 25 \\
\hline 8 & 9 & 37 & 30 & 9 & 8 & 9 & 38 & 21 & 25 & 8 & 9 & 39 & 8 & 49 \\
\hline 8 & 11 & 32 & 55 & 9 & 8 & 11 & 59 & 28 & 9 & $/$ & $/$ & $/$ & $/$ & $/$ \\
\hline
\end{tabular}

Table 7: Integral graphs $S_{6}(m, n, t)$.

Proof. For (3), by Theorem 3.2 (6.3), the necessary and sufficient condition for $S_{6}(m, n, 0)$ to be an integral graph is that there are positive integers $a$ and $b$ satisfying

$$
\left\{\begin{array}{cl}
a^{2}+b^{2} & =2 m+2 n+2, \\
a^{2} b^{2} & =2 n(2 m+1) .
\end{array}\right.
$$

Let $(2 m+1,2 n)=d$. By $(27)$ we have

$$
2 m+1=d m_{1}^{2}, \quad 2 n=d n_{1}^{2}, \quad a b=d m_{1} n_{1},
$$

where $m_{1}$ and $n_{1}$ are positive integers, and $\left(m_{1}, n_{1}\right)=1$. By using (27) and (28), we get

$$
(a+b)^{2}-d\left(m_{1}+n_{1}\right)^{2}=1 .
$$

Clearly, if $d$ is a perfect square, then the Diophantine equation (29) has no integral solutions.

Let $d$ be a positive integer but not a perfect square. Then Eq.(29) is a Pell equation. Let $\varepsilon=a_{1}+b_{1} \sqrt{d}$ be the fundamental solution of Eq.(2). By (29), we deduce as before that

$$
a+b=\frac{\varepsilon^{k}+\bar{\varepsilon}^{k}}{2}, \quad m_{1}+n_{1}=\frac{\varepsilon^{k}-\bar{\varepsilon}^{k}}{2 \sqrt{d}}, \quad k>0,
$$

where $\bar{\varepsilon}=a_{1}-b_{1} \sqrt{d}$ and $\varepsilon \bar{\varepsilon}=1$.

In view of (30) and $a b=2 d m_{1} n_{1}$ (see (28)), we get

$$
\left(2 b-\frac{\varepsilon^{k}+\bar{\varepsilon}^{k}}{2}\right)^{2}-d\left(2 n_{1}-\frac{\varepsilon^{k}-\bar{\varepsilon}^{k}}{2 \sqrt{d}}\right)^{2}=1 .
$$


Thus, we have

$$
2 b=\frac{\varepsilon^{k}+\bar{\varepsilon}^{k}}{2}+\frac{\varepsilon^{l}+\bar{\varepsilon}^{l}}{2}, \quad 2 n_{1}=\frac{\varepsilon^{k}-\bar{\varepsilon}^{k}}{2 \sqrt{d}}+\frac{\varepsilon^{l}-\bar{\varepsilon}^{l}}{2 \sqrt{d}}, \quad l>0 .
$$

Hence,

$$
m_{1}=\left(\frac{\varepsilon^{k}-\bar{\varepsilon}^{k}}{2 \sqrt{d}}-\frac{\varepsilon^{l}-\bar{\varepsilon}^{l}}{2 \sqrt{d}}\right) / 2, \quad n_{1}=\left(\frac{\varepsilon^{k}-\bar{\varepsilon}^{k}}{2 \sqrt{d}}+\frac{\varepsilon^{l}-\bar{\varepsilon}^{l}}{2 \sqrt{d}}\right) / 2, \quad k>l>0 .
$$

Letting

$$
y_{n}=\frac{\varepsilon^{n}-\bar{\varepsilon}^{n}}{2 \sqrt{d}}, \quad n=0,1,2, \cdots,
$$

we obtain the Pell sequence (see [3])

$$
y_{0}=0, \quad y_{1}=b_{1}, \quad y_{n+2}=2 a_{1} y_{n+1}-y_{n}, \quad(n \geq 0) .
$$

Hence, all integral graphs $S_{6}(m, n, 0)$ (where $\left.1 \leq m<n\right)$ are given via

$$
2 m+1=d\left(\frac{y_{k}-y_{l}}{2}\right)^{2}, \quad 2 n=d\left(\frac{y_{k}+y_{l}}{2}\right)^{2}, \quad k>l>0,
$$

where $m$ and $n$ are positive integers.

The results in (1)-(2) and (4)-(8) can be proved similarly to Theorem 4.2 by using (6), (6.1)-(6.7) of Theorem 3.2.

For $S_{6}(m, n, t)$, when $t=0,1 \leq m<n$, we have obtained all integral graphs $S_{6}(m, n, 0)$. However, when $t=0,1 \leq n<m$, we have not found any such integral graph. So we raise the following question.

Question 4.9. Are there integral graphs $S_{6}(m, n, 0)$ with $1 \leq n<m$ ? Can we give a sufficient and necessary condition for $S_{6}(m, n, 0)(1 \leq n<m)$ to be an integral graph?

With similar arguments as before the following results are obtained by using Theorem $3.2(7)$.

Theorem 4.10. The graph $S_{8}(m, n)(m \geq 0, n \geq 0)$ is integral if and only if there exist nonnegative integers $a, b, c$ and $d$ such that $x^{4}-4 x^{3}-(m+n-5) x^{2}+(2 m+2 n-2) x+$ $m n-m-n$ and $x^{4}+4 x^{3}-(m+n-5) x^{2}-(2 m+2 n-2) x+m n-m-n$ can be factorized as $(x+a)(x-b)(x+c)(x-d)$ and $(x-a)(x+b)(x-c)(x+d)$, respectively, where $m$ and $n$ are nonnegative integers.

In particular, we have the following results for the graph $S_{8}(m, n)$.

(1) For $m=0$ the graph $S_{8}(0, n)=S_{8}(n, 0)$ is integral if and only if $n=k(k+2)$, where $k$ is a nonnegative integer.

(2) For $m=n$ the graph $S_{8}(n, n)$ is integral if and only if $n=k(k+1)$, where $k$ is a nonnegative integer. 


\begin{tabular}{|c|c|c|c|c|c||c|c|c|c|c|c|}
\hline$a$ & $b$ & $c$ & $d$ & $m$ & $n$ & $a$ & $b$ & $c$ & $d$ & $m$ & $n$ \\
\hline 6 & 8 & 9 & 11 & 50 & 98 & 11 & 13 & 13 & 15 & 147 & 192 \\
\hline 21 & 23 & 26 & 28 & 486 & 726 & 40 & 42 & 57 & 59 & 1682 & 3362 \\
\hline 44 & 46 & 51 & 53 & 2028 & 2700 & 47 & 49 & 50 & 52 & 2312 & 2592 \\
\hline 75 & 77 & 84 & 86 & 5780 & 7220 & $/$ & $/$ & $/$ & $/$ & $/$ & $/$ \\
\hline
\end{tabular}

Table 8: Integral graphs $S_{8}(m, n)=S_{8}(n, m)$.

(3) For $m<n$, let $a, b, c, d, m, n, t$ be as in Theorem 4.10, and given in Table 8, then the graph $S_{8}(m, n)=S_{8}(n, m)$ is integral.(Table 8 is obtained by computer search, where $0 \leq a \leq 100,0 \leq b \leq a+30, a \leq c \leq a+30, b \leq d \leq b+30$ and $0 \leq m<n$.)

The next statements are derived from Theorem 3.2 (8) -(11).

Theorem 4.11. The graph $S_{9}(m, n, p, q)(m, n, p, q \geq 1)$ is integral if and only if there exist positive integers $a, b$ and $c$ such that $x^{6}-(2 m+n+2 p+q+n q+1) x^{4}+(m+n+$ $m n+p+4 m p+2 n p+q+2 m q+2 n q+2 m n q+p q+2 n p q) x^{2}-(m p+n p+2 m n p+m q+$ $n q+2 m n q+2 m p q+2 n p q+4 m p q)$ can be factorized as $\left(x^{2}-a^{2}\right)\left(x^{2}-b^{2}\right)\left(x^{2}-c^{2}\right)$.

In particular, we have the following results for the graph $S_{9}(m, n, p, q)$.

(1) If $m=n=p=q$, then the graph $S_{9}(n, n, n, n)$ is integral if and only if $n=2 k^{2}$, where $k$ is a positive integer.

(2) If $m, n, p, q$ are not as in (1), and $a, b, c, m, n, p, q$ are as in Theorem 4.11, and given in Table 9 , then the graph $S_{9}(m, n, p, q)=S_{9}(p, q, m, n)$ is integral.(Table 9 is obtained by computer search, where $1 \leq a \leq 7, a \leq b \leq a+5, b \leq c \leq b+5$, and $m, n, p, q$ are not as in (1).)

\begin{tabular}{|c|c|c|c|c|c|c||c|c|c|c|c|c|c|}
\hline$a$ & $b$ & $c$ & $m$ & $n$ & $p$ & $q$ & $a$ & $b$ & $c$ & $m$ & $n$ & $p$ & $q$ \\
\hline 3 & 4 & 5 & 8 & 1 & 8 & 8 & 6 & 10 & 12 & 51 & 3 & 21 & 33 \\
\hline
\end{tabular}

Table 9: Integral graph $S_{9}(m, n, p, q)=S_{9}(p, q, m, n)$.

Theorem 4.12. The graph $S_{10}(n)$ is integral if and only if $n=k(k+2)$, where $k(\geq 0)$ is an integer.

\section{Theorem 4.13.}

(1) For $m \geq 1, n \geq 1$ the graph $S_{13}(m, n)$ is integral if and only if (i) $m=k(k+1)$ and $m(n+1)=l(l+1)$, where $k$ and $l$ are positive integers, or (ii) $n=1$, and $m=\frac{1}{2} k(k+1)$, where $k$ is a positive integer.

(2) For $m=n=k(k+1), k$ is a positive integer, then the graph $S_{13}(n, n)$ is integral. 
Theorem 4.14. The graph $S_{17}(m, n, p, q)$ is integral if and only if there exist positive integers $a, b$ and $c$ such that $x^{6}-(2 m+2 n+p+q+p q+1) x^{4}+[m(2+4 n+2 p+q+p q)+$ $\left.n+p+n p+2 n q+2 p q+2 n p q+p q^{2}\right] x^{2}-[2 m(n+p+n p+n q+p q+n p q)+2 n p q(q+1)]$ can be factorized as $\left(x^{2}-a^{2}\right)\left(x^{2}-b^{2}\right)\left(x^{2}-c^{2}\right)$, and one of the following two conditions holds: (i) $2 m$ is a perfect square, (ii) $q=1$, where $m, n, p$, $q$ are positive integers.

In particular, we have the following results for the graph $S_{17}(m, n, p, q)$.

(1) If $m=n$, then the graph $S_{17}(n, n, p, q)$ is integral if and only if $n=2 k^{2}$, and there exist nonnegative integers $a$ and $b$ such that $x^{4}-[2 n+(p+1)(q+1)] x^{2}+(q+1)[n(p+$ $1)+p(q+1)]$ can be factorized as $\left(x^{2}-a^{2}\right)\left(x^{2}-b^{2}\right)$, where $n, p, q$ and $k$ are positive integers.

(2) If $m=n=p=q$, then the graph $S_{17}(n, n, n, n)$ is integral if and only if $n=2 k^{2}$, where $k$ is a positive integer.

(3) If $m=n, p=1$, then the graph $S_{17}(n, n, 1, q)$ is integral if and only if $n=2 r^{2} s^{2} h^{2}$, $q=\left(r^{2}-s^{2}\right)^{2} h^{2}-1$, where $(r, s)=1, r>s, 2 \nmid r+s$, and $n, q, r, s, h$ are positive integers.

(4) If $m=n=2 k^{2} l^{2}, p=2 l^{2}$ and $q=k^{2}\left(2 l^{2}+1\right)-1$, then the graph $S_{17}(n, n, p, q)$ is integral.

(5) For $m=n$, let $a, b, m, n, p$ and $q$ be as in (1), and not as in (2)-(4), and given by Table 10. Then the graph $S_{17}(n, n, p, q)$ is integral. (Table 10 is obtained by computer search, where $1 \leq a \leq 40, a \leq b \leq a+20$, and $m, n, p$ and $q$ are not as in (2)-(4).)

(6) If $m \neq n, p=m, q=n$, then the graph $S_{17}(m, n, m, n)$ is integral if and only if there exist nonnegative integers $a$ and $b$ such that $x^{4}-(2 m+2 n+m n+1) x^{2}+2 m(n+1)^{2}$ can be factorized as $\left(x^{2}-a^{2}\right)\left(x^{2}-b^{2}\right)$, and one of the following two conditions holds: (i) $2 m$ and $n+m$ are perfect squares, (ii) $n=1, m+1$ is a perfect square, where $m$ and $n$ are positive integers.

(7) If $m=p=2, q=n$, then the graph $S_{17}(2, n, 2, n)$ is integral if and only if $n=l^{2}-2$ and $2 n+2=k(k+1)$, where $n, l$ and $k$ are positive integers. (Examples are presented in Table 11. Table 11 is obtained by computer search, where $1 \leq k \leq 10000$.)

(8) For $m \neq n$, let $a, b, c, m, n, p, q$ be as in Theorem 4.14, and given in Table 12. Then the graph $S_{17}(m, n, p, q)$ is integral.(Table 12 is obtained by computer search, where $1 \leq a \leq 13, a \leq b \leq a+5, b \leq c \leq b+10$, and $m \neq n$.)

\begin{tabular}{|c|c|c|c|c|c||c|c|c|c|c|c|}
\hline$a$ & $b$ & $m$ & $n$ & $p$ & $q$ & $a$ & $b$ & $m$ & $n$ & $p$ & $q$ \\
\hline 8 & 18 & 50 & 50 & 11 & 23 & 14 & 34 & 288 & 288 & 193 & 3 \\
\hline 16 & 36 & 200 & 200 & 11 & 95 & $/$ & $/$ & $/$ & $/$ & $/$ & $/$ \\
\hline
\end{tabular}

Table 10: Integral graph $S_{17}(n, n, p, q)$. 


\begin{tabular}{|c|c|c||c|c|c||c|c|c|}
\hline$n$ & $l$ & $k$ & $n$ & $l$ & $k$ & $n$ & $l$ & $k$ \\
\hline 2 & 2 & 2 & 14 & 4 & 5 & 119 & 11 & 15 \\
\hline 527 & 23 & 32 & 4094 & 64 & 90 & 17954 & 134 & 189 \\
\hline 139127 & 373 & 527 & 609959 & 781 & 1104 & 4726274 & 2174 & 3074 \\
\hline 20720702 & 4552 & 6437 & $/$ & $/$ & $/$ & $/$ & $/$ & $/$ \\
\hline
\end{tabular}

Table 11: Integral graphs $S_{17}(2, n, 2, n)$.

\begin{tabular}{|c|c|c|c|c|c|c||c|c|c|c|c|c|c|}
\hline$a$ & $b$ & $c$ & $m$ & $n$ & $p$ & $q$ & $a$ & $b$ & $c$ & $m$ & $n$ & $p$ & $q$ \\
\hline 4 & 5 & 6 & 2 & 14 & 2 & 14 & 5 & 5 & 6 & 2 & 16 & 1 & 24 \\
\hline 7 & 9 & 10 & 18 & 48 & 1 & 48 & 9 & 10 & 11 & 18 & 52 & 1 & 80 \\
\hline 11 & 11 & 12 & 8 & 64 & 1 & 120 & 11 & 14 & 15 & 50 & 100 & 1 & 120 \\
\hline 11 & 15 & 16 & 2 & 119 & 2 & 119 & 13 & 14 & 15 & 18 & 108 & 1 & 168 \\
\hline 13 & 15 & 16 & 32 & 124 & 1 & 168 & $/$ & $/$ & $/$ & $/$ & $/$ & $/$ & $/$ \\
\hline
\end{tabular}

Table 12: Integral graphs $S_{17}(m, n, p, q)$.

Proof. (i) Similar to the proof of Theorem 4.2, this theorem and the statements in (1), (2), (4)-(8) are proven by Theorem 3.2 (11), (11.1)-(11.5).

(ii) Next we shall prove (3). By (11.3) of Theorem 3.2, the graph $S_{17}(n, n, 1, q)$ is integral if and only if $2 n, q+1$ and $2 n+q+1$ are perfect squares. Assume that $n=2 k^{2} h^{2}$, $q+1=l^{2} h^{2}$ and $2 n+q+1=t^{2} h^{2}$, where $k, l, t, h$ are positive integers, and $(l, 2 k)=1$. Then we get

$$
l^{2}+(2 k)^{2}=t^{2} .
$$

Lemma 2.11 yields $l=r^{2}-s^{2}, 2 k=2 r s, t=r^{2}+s^{2}, n=2 r^{2} s^{2} h^{2}, q=\left(r^{2}-s^{2}\right)^{2} h^{2}-1$, where $(r, s)=1, r>s>0,2 \nmid r+s$, and $n, q, r, s, h$ are positive integers.

By (7) of Theorem 4.14, we see that the graph $S_{17}(2, n, 2, n)$ is integral if and only if $n=l^{2}-2$ and $2 l^{2}-2=k(k+1)$, where $n, l$ and $k$ are positive integers. Hence, we raise the following question.

Question 4.15. What are all positive integral solutions of the Diophantine equation $2 l^{2}-$ $2=k(k+1)$ ?

We finally list the results obtained from Theorem $3.2(12)-(15)$.

Theorem 4.16. The graph $S_{18}(n, p, q, t)$ is integral if and only if there exist nonnegative integers $a, b, c, d$, $e$ and $f$ such that $x^{6}-4 x^{5}-(2 n+p+q+t-6) x^{4}+(6 n+2 p+2 q+4 t-4) x^{3}-$ $(6 n+p-n p+q-n q-p q+6 t-p t-q t-1) x^{2}+(2 n-n p-n q+4 t-2 p t-2 q t) x-t(p-1)(q-1)$ can be factorized as $(x+a)(x+b)(x+c)(x-d)(x-e)(x-f)$, and one of the following two conditions holds: (i) $t$ is a perfect square, (ii) $n=1$, where $n \geq 1, p$, $q$ and $t$ are nonnegative integers.

In particular, we have the following results for the graph $S_{18}(n, p, q, t)$. 
(1) For $p=q$ the graph $S_{18}(n, p, p, t)$ is integral if and only if there exist nonnegative integers $a, b, c$ and $d$ such that $x^{4}-2 x^{3}-(p+t+2 n-1) x^{2}+2(n+t) x+t(p-1)$ can be factorized as $(x+a)(x-b)(x+c)(x-d)$, and one of the following two conditions holds: (i) $p$ and $t$ are perfect squares, (ii) $n=1$, and $p$ is a perfect square, where $n$ is a positive integer, and $p, t$ are nonnegative integers.

(2) For $p=q=t=1$ the graph $S_{18}(n, 1,1,1)$ is integral if and only if $n=\frac{1}{2} k(k+1)-1$, where $k(\geq 2)$ is a positive integer.

(3) For $p=q=t=0$ the graph $S_{18}(n, 0,0,0)=S_{5}(n, n)$ is integral if and only if $n=\frac{1}{2} k(k+1)$, where $k$ is a positive integer.

(4) For $n=2 t, p=q=t=k^{2}$ the graph $S_{18}\left(2 k^{2}, k^{2}, k^{2}, k^{2}\right)$ is integral if and only if $k$ is a positive integer satisfying the Pell equation

$$
l^{2}-8 k^{2}=1 .
$$

Proof. Similar to the proof of Theorem 4.2, we easily check the correctness of this theorem and the results in (1), (2) and (3) by using Theorem 3.2 (12).

Next we prove (4). By Theorem $3.2(12.4)$, we see that the graph $S_{18}\left(2 k^{2}, k^{2}, k^{2}, k^{2}\right)$ is integral if and only if there are positive integers $k, r$ and $s$ such that

$$
\left\{\begin{array}{l}
k(k-1)=r(r+2 k+1), \\
k(k+1)=s(s+2 k-1) .
\end{array}\right.
$$

This relation yields

$$
(2 k+r+s)(s-r-1)=0 \quad \text { and } \quad s^{2}+(2 k-1) s-k(k+1)=0 .
$$

Then $s=r+1$, and $s=\frac{-(2 k-1) \pm \sqrt{8 k^{2}+1}}{2}$.

Hence, $s$ is a positive integer if and only if $8 k^{2}+1$ is a perfect square. Let $8 k^{2}+1=l^{2}$, then $k$ is a positive integer satisfying the Pell equation (31). All positive integral solutions of (31) are given by

$$
l+k \sqrt{8}=u_{n}+v_{n} \sqrt{8}=(3+\sqrt{8})^{n},
$$

where $n=1,2, \cdots$.

Thus, the proof is complete.

Theorem 4.17. The graph $S_{19}(m, n, p, t)$ is integral if and only if $\left(x^{2}-t\right)^{n(m-1)}\left[x^{4}-\right.$ $\left.(m+t+p+1) x^{2}+m+t+p t\right]^{n-1}\left\{x^{6}-(m+n+m n+p+n p+t+1) x^{4}+[m+n+m n+\right.$ $\left.\left.m n^{2}-2 n p+2 m n p+m n^{2} p+n p^{2}+t(1+n+p+n p)\right] x^{2}-n(p-1)^{2}(m n+t)\right\}=0$ has only integral roots, where $m(\geq 1), n(\geq 1), p(\geq 1), t(\geq 0)$ are integers.

In particular, we have the following results for the graph $S_{19}(m, n, p, t)$.

(1) If $p=1$, then the graph $S_{19}(m, n, 1, t)$ is integral if and only if $\left(x^{2}-t\right)^{n(m-1)}\left[x^{4}-\right.$ $\left.(m+t+2) x^{2}+m+2 t\right]^{n-1}\left[x^{4}-(m+2 n+m n+t+2) x^{2}+(n+1)(m+2 m n+2 t)\right]=0$ has only integral roots, where $m(\geq 1), n(\geq 1)$ and $t(\geq 0)$ are integers. 
(2) If $m=2, p=t=1$, then the graph $S_{19}(2, n, 1,1)$ is integral if and only if $n=$ $\frac{1}{2} k(k+1)-1$, where $k(\geq 2)$ is a positive integer.

Theorem 4.18. The graph $S_{20}(n, p, q)$ is integral if and only if $(i) n=k(k+1)$ and $p q=(l+k+1)(l-k)$, or (ii) $p=1, n=r$ and $q=s(s+1)-r \geq 1$, where $n, p, q, l, k$, $r$ and $s$ are positive integers, and $l>k$.

Corollary 4.19. If the graph $S_{20}(k(k+1), p, q)$ is integral, then the graph $S_{20}(k(k+1), q, p)$ is integral too.

Theorem 4.20. The graph $S_{21}(m, t)$ is integral if and only if $(i) t=k(k+1)$ and $m=(l+k+1)(l-k)$, or (ii) $m=1$ and $t=r(r+1)-1$, where $m(\geq 1), t(\geq 0), l$ $(\geq 1), k(\geq 0)$ and $r(\geq 1)$ are integers, and $l>k$.

\section{Further discussion}

In the present paper, we have mainly investigated the 15 nonregular bipartite integral graphs $S_{1}, S_{2}, S_{3}, S_{4}, S_{5}, S_{6}, S_{8}, S_{9}, S_{10}, S_{13}, S_{17}, S_{18}, S_{19}, S_{20}$ and Figure 5 (i.e. $S_{21}$ ) of [2]. Fifteen classes of larger integral graphs were constructed based on the structures of these integral graphs. These classes are connected nonregular and bipartite graphs except for several disconnected graphs for which one or several of the parameters are zero. However, we have not found appropriate methods to construct new integral graphs from the graphs $S_{7}, S_{11}, S_{12}, S_{14}, S_{15}, S_{16}$ of [2] or Theorem 3.1. Thus, we raise the following question.

Question 5.1. Can we construct new integral graphs from the graphs $S_{7}, S_{11}, S_{12}, S_{14}$, $S_{15}, S_{16}$ of [2] or Theorem 3.1?

Although we obtained fifteen new classes of integral graphs from the graphs $S_{1}-S_{6}$, $S_{8}-S_{10}, S_{13}, S_{17}-S_{21}$ in Theorem 3.1 or [2], we think that other methods can be found to construct new integral graphs. For example, let $K_{n}^{t}$ be obtained by joining $t$ new end vertices to each vertex of $K_{n}$, then the graph $K_{n(n+1)}^{n(n+1)}$ is integral (see [10]). We note that the graph $K_{n}^{t}$ can be constructed from $S_{1}=K_{1,4}$. Hence, we raise the following question.

Question 5.2. How to construct new integral graphs from the graphs $S_{1}, S_{2}, S_{3}, S_{4}, S_{5}$, $S_{6}, S_{8}, S_{9}, S_{10}, S_{13}, S_{17}, S_{18}, S_{19}, S_{20}, S_{21}$ in Theorem 3.1 or [2]?

For the graphs $S_{1}(t)=K_{1, t}, S_{2}(n, t), S_{3}(m, n, t), S_{5}(m, n), S_{10}(n), S_{13}(m, n), S_{20}(n$, $p, q), S_{21}(m, t)$, in fact, we have given a necessary and sufficient integrality condition. However, it is very difficult to find all integral graphs of the type $S_{4}(m, n, p, q), S_{6}(m, n, t)$, $S_{8}(m, n), S_{9}(m, n, p, q), S_{17}(m, n, p, q), S_{18}(n, p, q, t), S_{19}(m, n, p, t)$. Hence, we come to

Question 5.3. Can we give a better necessary and sufficient condition for the above 7 classes of graphs to be integral? 
Note that in connection with Question 5.3, in the present paper, we found some results for the above 7 classes of graphs by computer search and number theory. It was proved that the problem of finding such integral graphs is equivalent to the problem of solving some Diophantine equations. Finally we ask

Question 5.4. What are all positive integral solutions for these Diophantine equations, for example for the Diophantine equations (13)-(20), (23), and so on?

\section{Acknowledgements}

The authors would like to thank Professor Xueliang Li for many useful discussions and suggestions in this work.

\section{References}

[1] K. T. Balińska, D. Cvetković, Z. Radosavljević, S. K. Simić, D. Stevanović, A survey on integral graphs, Univ. Beograd. Publ. Elektrotehn. Fak. Ser. Mat. 13 (2002) 42-65.

[2] K. T. Balińska and S. K. Simić, The nonregular, bipartite, integral graphs with maximum degree 4. Part I: Basic properties, Discrete Math. 236 (1-3) (2001) 13-24.

[3] Z.F. Cao, Integral trees of diameter $R(3 \leq R \leq 6)$ (in Chinese), Heilongjiang Daxue Ziran Kexue Xuebao (2) (1988) 1-3, 95.

[4] Z.F. Cao, Introductory Diophantine Equations (in Chinese), Haerbin Polytechnical University Press (1989).

[5] D. Cvetković, M. Doob and H. Sachs, Spectra of Graphs-Theory and Application, Academic Press, New York, Francisco, London (1980).

[6] P.J. Davis, Circulant Matrices, John Wiley \& Sons, New York (1979).

[7] F. Harary and A.J. Schwenk, Which graphs have integral spectra?, In: R. Bari and F. Harary (Eds.), Graphs and Combinatorics, Lecture Notes in Mathematics 406, Springer-Verlag, Berlin (1974) 45-51.

[8] C.D. Pan and C.B. Pan, Elementary Number Theory (in Chinese), Peking University Press, Beijing (1994).

[9] L.G. Wang and X.L. Li, Some new classes of integral trees with diameters 4 and 6, Australas. J. Combin. 21 (2000) 237-243.

[10] L.G. Wang, X.L. Li and S.G. Zhang, Construction of integral graphs, Appl. Math. J. Chinese Univ. Ser. B 15 (3) (2000) 239-246.

[11] L.G. Wang, X.L. Li and S.G. Zhang, Families of integral trees with diameters 4, 6 and 8, Discrete Appl. Math. 136 (2-3) (2004) 349-362. 